\title{
Perturbations of superstable linear hyperbolic systems
}

\author{
I. Kmit * N. Lyul'ko ${ }^{\dagger}$
}

\begin{abstract}
The paper deals with initial-boundary value problems for linear non-autonomous first order hyperbolic systems whose solutions stabilize to zero in a finite time. We prove that problems in this class remain exponentially stable in $L^{2}$ as well as in $C^{1}$ under small bounded perturbations. To show this for $C^{1}$, we prove a general smoothing result implying that the solutions to the perturbed problems become eventually $C^{1}$-smooth for any $L^{2}$-initial data.
\end{abstract}

Key words: first order hyperbolic systems, smoothing boundary conditions, superstability, exponential stability, bounded perturbations, evolution family

Mathematics Subject Classification: 35B20, 35B35, 35B40, 35B45, 35B65, 35L50

\section{Introduction}

A linear system

$$
\frac{d}{d t} x(t)=A(t) x(t), \quad x(t) \in X \quad(0 \leq t \leq \infty),
$$

on a Banach space $X$ is called exponentially stable if there exist positive reals $\gamma$ and $M=M(\gamma)$ such that every solution $x(t)$ satisfies the estimate

$$
\|x(t)\| \leq M e^{-\gamma t}\|x(0)\|, \quad t \geq 0
$$

where $\|\cdot\|$ denotes the norm in $X$.

The papers $[3,4,8]$ address a stronger property of exponentially stable systems, known as superstability. They consider the Cauchy problem for the autonomous version of (1), where $A(t)=A$ does not depend on $t$. Moreover, $A: X \rightarrow X$ is supposed to be the infinitesimal generator of a strongly continuous semigroup $T(t)$; see [14, 27]. A semigroup $T(t)$ is called superstable $[3,4,23,29]$ if its stability index is $-\infty$, that is

$$
\lim _{t \rightarrow \infty} \frac{\log \|T(t)\|}{t}=-\infty .
$$

*Institute of Mathematics, Humboldt University of Berlin. On leave from the Institute for Applied Problems of Mechanics and Mathematics, Ukrainian National Academy of Sciences. E-mail: kmit@mathematik.hu-berlin.de

${ }^{\dagger}$ Sobolev Institute of Mathematics, Russian Academy of Sciences and Novosibirsk State University, Russia. E-mail: natlyl@mail.ru 
In this case the system (1) is called superstable also. The superstability property implies that the system is exponentially stable and, moreover, the estimate (2) holds for every $\gamma>0$. For a superstable system the resolvent $R(\lambda ; A)$ of the operator $A$, which is defined by the formula

$$
R(\lambda ; A) x=\int_{0}^{\infty} e^{-\lambda t} T(t) x d t, \quad x \in X
$$

is an entire function of the complex parameter $\lambda$, and the spectrum of the operator $A$, which we denote by $\sigma(A)$, is empty. The superstability property makes sense only for systems in infinite-dimensional Banach spaces, since any linear operator $A: X \rightarrow X$ in a finite-dimensional space $X$ has a non-empty point spectrum, and the stability index is equal to the maximum of the real parts of the eigenvalues of $A$.

An important subclass of superstable systems, that will be studied in the present paper, consists of the systems whose solutions stabilize to zero after some time. The time of the stabilization is called a finite time extinction. The simplest example is given by the initial-boundary value problem [14]

$$
\begin{array}{ll}
u_{t}+u_{x}=0, & (x, t) \in(0,1) \times(0, \infty), \\
u(x, 0)=u_{0}(x), & x \in[0,1], \\
u(0, t)=0, & t \in(0, \infty) .
\end{array}
$$

It is easy to check that all solutions to this problem stabilize to zero for $t>1$. Similar examples for the wave equation are given in [7, 19, 24].

Here we address superstable initial-boundary value problems with finite time extinction for linear non-autonomous hyperbolic systems. We consider bounded perturbations of such problems and investigate the asymptotic behavior of their solutions. In contrast to the autonomous case, which is well-studied, the non-autonomous case has been considered in the literature only episodically.

The recent papers $[25,26,28,31,32,33]$ are devoted to superstable hyperbolic models intensively used in the control theory. By introducing control parameters in the boundary conditions and/or in the coefficients of the differential equations, such systems can be stabilized to a desired state in a finite time, which, from the physical point of view, is even more preferable than the infinite time stabilization. Superstable hyperbolic systems are usually supplemented with the so-called quiet boundaries [32], where the influence of the reflected waves is minimized or even neglected. Mathematically, the quiet boundaries are described by means of the so-called smoothing boundary conditions, that also will be considered in the present paper.

The paper is organized as follows. In Section 2 we state the problem and formulate our results about the existence of evolution families, smoothing properties of solutions, and exponential stability of solutions. Some comments and examples related to applications are given in Section 3. In particular, Example 3.4 shows how our results obtained for linear systems can be applied to show the exponential stability of solutions to nonlinear problems. Using a priori estimates, in Section 4 we prove that the problem under consideration generates an evolution family on $L^{2}(0,1)^{n}$. In Section 4.3 we extend the results of $[10,16,17,21]$ by showing that boundary operators of reflection type cause the smoothing effect in the sense that the solutions reach the $C^{1}$-regularity for any $L^{2}$-initial data 
after some time. Furthermore, we provide general conditions for that. We also discuss the relationship between the smoothing property and the stabilization to zero. Finally, in Section 5.2, using the variation of constants formula, we prove that superstable hyperbolic operators remain exponentially stable under small bounded perturbations.

Previous work Our stability analysis shows that the exponential decay rate $\gamma$ of solutions to the perturbed problems can be arbitrary large provided the perturbations are sufficiently small. Similar questions are addressed for the wave equation perturbed by velocity damping, see e.g. $[12,13]$ and references therein.

In [14] the authors investigate a relationship between the asymptotic behavior of the spectrum and the resolvent of an operator $A$ and the stability of the corresponding semigroup. In [20], properties of the resolvent $R(\lambda ; A)$ are used to establish a criterion that a strongly continuous semigroup $T(t)$ is eventually vanishing or, the same, is nilpotent [8]. Stability of nilpotent semigroups is investigated in [5]. Properties of the strongly continuous semigroup $T(t)$ generated by $A$ are used in [8] for giving criteria that the autonomous system (1) is asymptotically stable, superstable, or stabilizes to zero in a finite time.

In [10] the second author considers initial-boundary value problems of the type (1) for autonomous strictly hyperbolic systems. Analyzing the resolvent of $A$, she describes a class of boundary conditions for which solutions eventually stabilize to zero. The condition $\sigma(A)=\emptyset$ gives a criterion for stabilization for a decoupled hyperbolic system. It is also shown that the stabilization property is closely related to increasing smoothness of solutions to the perturbed autonomous problems. In the autonomous strictly hyperbolic case the smoothing effect is addressed in $[10,21,22]$, while in the non-autonomous weakly hyperbolic case it is investigated in $[16,17]$.

A comprehensive review of the available results on asymptotic behavior of solutions to linear and quasi-linear hyperbolic problems can be found in $[2,6,13]$.

\section{Problem setting and the main results}

Notation Set $\Pi=\{(x, t): 0<x<1, t \in \mathbb{R}\}$. Let $B C(\Pi)$ (respectively, $B C(\mathbb{R})$ ) denote the Banach space of all bounded and continuous maps $u: \bar{\Pi} \rightarrow \mathbb{R}$ (respectively, $u: \mathbb{R} \rightarrow \mathbb{R}$ ), with the norm

$$
\left.\|u\|_{\infty}=\sup _{(x, t) \in \Pi}|u| \quad \text { (respectively, }\|u\|_{\infty}=\sup _{t \in \mathbb{R}}|u|\right)
$$

Furthermore, let $B C^{1}(\Pi)$ denote the Banach space of all $u \in B C(\Pi)$ such that $\partial_{x} u \in$ $B C(\Pi)$ and $\partial_{t} u \in B C(\Pi)$, with the norm

$$
\|u\|_{1}=\|u\|_{\infty}+\left\|\partial_{x} u\right\|_{\infty}+\left\|\partial_{t} u\right\|_{\infty} .
$$

If $X$ is a Banach space, then the $n$-th Cartesian power $X^{n}$ is considered a Banach space with the norm

$$
\|u\|_{X^{n}}=\max _{i \leq n}\left\|u_{i}\right\|_{X}
$$


As usual, by $\mathcal{L}(X, Y)$ we denote the space of linear bounded operators from a Banach space $X$ into a Banach space $Y$, and write $\mathcal{L}(X)$ for $\mathcal{L}(X, X)$.

Problem setting $\quad$ In the strip $\Pi$ we consider the following decoupled non-autonomous hyperbolic system:

$$
\partial_{t} u+a(x, t) \partial_{x} u+b_{d}(x, t) u=0, \quad(x, t) \in \Pi,
$$

and its perturbed version

$$
\partial_{t} u+a(x, t) \partial_{x} u+\left(b_{d}(x, t)+\tilde{b}(x, t)\right) u=0, \quad(x, t) \in \Pi,
$$

where $n \geq 2, u=\left(u_{1}, \ldots, u_{n}\right)$ is a vector of real-valued functions, $a=\operatorname{diag}\left(a_{1}, \ldots, a_{n}\right)$ and $b_{d}=\operatorname{diag}\left(b_{1}, \ldots, b_{n}\right)$ are diagonal matrices, and $\tilde{b}$ is an $(n \times n)$-matrix with entries $\tilde{b}_{j k}$. The systems $(2.1)$ and (2.2) will be endowed with the reflection boundary conditions

$$
\begin{array}{ll}
u_{j}(0, t)=\sum_{k=1}^{m} p_{j k} u_{k}(1, t)+\sum_{k=m+1}^{n} p_{j k} u_{k}(0, t), \quad 1 \leq j \leq m, \\
u_{j}(1, t)=\sum_{k=1}^{m} p_{j k} u_{k}(1, t)+\sum_{k=m+1}^{n} p_{j k} u_{k}(0, t), \quad m<j \leq n,
\end{array}
$$

where the integer $m$ is fixed in the range $0 \leq m \leq n$ and the reflection coefficients $p_{i j}$ are real constants.

Suppose that

$$
a_{j}, b_{j}, \tilde{b}_{j k} \in B C^{1}(\Pi) \text { for all } j \leq n
$$

and

$$
\inf _{(x, t) \in \bar{\Pi}} a_{j} \geq \Lambda_{0} \text { for all } j \leq m \text { and } \quad \sup _{(x, t) \in \bar{\Pi}} a_{j} \leq-\Lambda_{0} \text { for all } j>m
$$

for some $\Lambda_{0}>0$. Condition (2.5) ensures that the hyperbolic systems (2.1) and (2.2) are non-degenerate and that all their characteristics are uniformly bounded in $\bar{\Pi}$.

Given $\tau \in \mathbb{R}$, we also consider the initial conditions at $t=\tau$, namely

$$
u(x, \tau)=\varphi(x), \quad x \in[0,1] .
$$

Set

$$
\Pi_{\tau}=\{(x, t): 0<x<1, t>\tau\} .
$$

We are interested in the long time behavior of solutions to the perturbed problem (2.2), (2.6), (2.3) in $\Pi_{\tau}$, under the assumption that solutions to the unperturbed problem (2.1), (2.6), (2.3) in $\Pi_{\tau}$ stabilize to zero in a finite time. We will formulate our results in terms of evolution families generated by the unperturbed and the perturbed problems; this concept will be introduced below. In fact, we will prove the existence of an evolution family for the general linear first order hyperbolic system

$$
\partial_{t} u+a(x, t) \partial_{x} u+b(x, t) u=0, \quad(x, t) \in \Pi,
$$


subjected to the boundary conditions (2.3). We assume that $b$ is an $(n \times n)$-matrix with entries

$$
b_{i j} \in B C^{1}(\Pi) \quad \text { for all } i, j \leq n .
$$

The well-posedness of the problem (2.7), (2.6), (2.3) in $\Pi_{\tau}$ in the spaces of continuous and continuously differentiable functions is investigated in $[1,15]$.

Characteristics and integral representation For given $j \leq n, x \in[0,1]$, and $t \in \mathbb{R}$, the $j$-th characteristic of (2.7) passing through the point $(x, t)$ is defined as the solution $\xi \in[0,1] \mapsto \omega_{j}(\xi, x, t) \in \mathbb{R}$ to the initial value problem

$$
\partial_{\xi} \omega_{j}(\xi, x, t)=\frac{1}{a_{j}\left(\xi, \omega_{j}(\xi, x, t)\right)}, \omega_{j}(x, x, t)=t
$$

The assumption (2.5) implies that, if $(x, t) \in \bar{\Pi}_{\tau}$, then the characteristic curve $\theta=$ $\omega_{j}(\xi, x, t)$ reaches the boundary of $\Pi_{\tau}$ in two points with distinct ordinates. Let $x_{j}(x, t)$ denote the abscissa of that point whose ordinate is smaller. The condition (2.5) ensures that the value of $x_{j}(x, t)$ does not depend on $x, t$ if $t>\tau+\frac{1}{\Lambda_{0}}$. Therefore, $x_{j}(x, t)$ in this range takes on the constant value

$$
x_{j}= \begin{cases}0 & \text { if } 1 \leq j \leq m \\ 1 & \text { if } m<j \leq n\end{cases}
$$

Let

$$
c_{j}(\xi, x, t)=\exp \int_{x}^{\xi}\left(\frac{b_{j j}}{a_{j}}\right)\left(\eta, \omega_{j}(\eta, x, t)\right) d \eta, \quad d_{j}(\xi, x, t)=\frac{c_{j}(\xi, x, t)}{a_{j}\left(\xi, \omega_{j}(\xi, x, t)\right)} .
$$

We define a linear bounded operator $P \in \mathcal{L}\left(B C(\Pi)^{n}, B C(\mathbb{R})^{n}\right)$ by

$$
(P u)_{j}(t)=\sum_{k=1}^{m} p_{j k} u_{k}(1, t)+\sum_{k=m+1}^{n} p_{j k} u_{k}(0, t), \quad j \leq n .
$$

Straightforward calculations show that a $C^{1}$-map $u: \bar{\Pi}_{\tau} \rightarrow \mathbb{R}^{n}$ is a solution to the problem $(2.7),(2.6),(2.3)$ if and only if it satisfies the following system of integral equations

$$
\begin{aligned}
& u_{j}(x, t)=(Q u)_{j}(x, t) \\
& \quad-\int_{x_{j}(x, t)}^{x} d_{j}(\xi, x, t) \sum_{k \neq j} b_{j k}\left(\xi, \omega_{j}(\xi, x, t)\right) u_{k}\left(\xi, \omega_{j}(\xi, x, t)\right) d \xi, \quad j \leq n,
\end{aligned}
$$

where the affine operator $Q$ is defined by

$(Q u)_{j}(x, t)= \begin{cases}c_{j}\left(x_{j}(x, t), x, t\right)(P u)_{j}\left(\omega_{j}\left(x_{j}(x, t), x, t\right)\right) & \text { if } x_{j}(x, t)=0 \text { or } x_{j}(x, t)=1 \\ c_{j}\left(x_{j}(x, t), x, t\right) \varphi_{j}\left(x_{j}(x, t)\right) & \text { if } \quad x_{j}(x, t) \in(0,1)\end{cases}$

on a subset of $C\left(\bar{\Pi}_{\tau}\right)^{n}$ of functions satisfying the initial condition (2.6). A continuous function $u$ satisfying $(2.12)$ in $\bar{\Pi}_{\tau}$ is called a continuous solution to $(2.7),(2.6),(2.3)$. 
Our results In Section 3 we prove that the problem $(2.7),(2.6),(2.3)$, where $\tau \in \mathbb{R}$ is an arbitrary fixed initial time, generates an evolution family $\{U(t, \tau)\}_{t \geq \tau}$ mapping an initial function $\varphi$ given at time $\tau$ into the solution $U(t, \tau) \varphi$ of the problem (2.7), (2.6), (2.3) at time $t$. We now define this concept formally.

Definition 2.1 [27] A two-parameter family $\{U(t, \tau)\}_{t \geq \tau}$ of linear bounded operators on a Banach space $X$ is called an evolution family if it satisfies the following properties:

- $U(\tau, \tau)=I$ and $U(t, s) U(s, \tau)=U(t, \tau)$ for all $t \geq s \geq \tau$;

- the map $(t, \tau) \in \mathbb{R}^{2} \rightarrow U(t, \tau) \in \mathcal{L}(X)$ is strongly continuous for all $t \geq \tau$.

Definition 2.2 [27] An evolution family $\{U(t, \tau)\}_{t \geq \tau}$ on a Banach space $X$ is called exponentially bounded if there exist $\omega \in \mathbb{R}$ and $M=M(\omega) \geq 1$ such that

$$
\|U(t, \tau)\|_{\mathcal{L}(X)} \leq M e^{\omega(t-\tau)} \text { for all } t \geq \tau .
$$

An evolution family is uniformly exponentially stable if $\omega<0$.

We are now prepared to state our first result.

Theorem 2.3 Suppose that the coefficients in the system (2.7) fulfill the conditions (2.4), (2.5), and (2.8). Then the problem (2.7), (2.3) generates an exponentially bounded evolution family $\{U(t, \tau)\}_{t \geq \tau}$ on the space $L^{2}(0,1)^{n}$.

Our second result, Theorem 2.5 below, states that the evolution family has a smoothing property of the following kind.

Definition 2.4 Let $Y \hookrightarrow Z$ be continuously embedded Banach spaces and, for each $\tau$ and $t \geq \tau, V(t, \tau) \in \mathcal{L}(Z)$. The two-parameter family $\{V(t, \tau)\}_{t \geq \tau}$ is called smoothing from $Z$ to $Y$ if there is $T>0$ such that $V(t, \tau) \in \mathcal{L}(Z, Y)$ for all $t \geq \tau+T$.

From (2.12) and (2.13) it follows that, if the system (2.7) is decoupled (non-diagonal elements of $b$ vanish), then the continuous function $u$ fulfilling the equation

$$
u(x, t)=(Q u)(x, t)
$$

is a continuous solution to the problem $(2.7),(2.3),(2.6)$. Let us introduce a linear bounded operator $C \in \mathcal{L}\left(B C(\mathbb{R})^{n}, B C(\Pi)^{n}\right)$ by

$$
(C v)_{j}(x, t)=c_{j}\left(x_{j}, x, t\right) v_{j}\left(\omega_{j}\left(x_{j}, x, t\right)\right), \quad j \leq n,
$$

where $x_{j}$ is given by (2.10). Therefore, every continuous solution to the decoupled problem stabilizes to zero in a finite time $d>0$ if and only if

$$
\text { there is } k \in \mathbb{N} \text { such that }(C P)^{k} u \equiv 0 \text { for all } u \in C(\bar{\Pi})^{n} \text {. }
$$

Moreover, due to (2.5), the time of extinction does not exceed $\frac{k}{\Lambda_{0}}$. The condition $(2.14)$ will be crucial for the following smoothing result, that will be proved in Section 4.3. 
Theorem 2.5 Assume that the coefficients $a_{j}, b_{j k}$, and $p_{j k}$ in (2.7) and (2.3) fulfill the conditions (2.4), (2.5), (2.8), and (2.14). Moreover, assume that

$$
\text { for all } 1 \leq j \neq k \leq n \text { there exists } \beta_{j k} \in B C^{1}(\Pi) \text { such that } b_{j k}=\beta_{j k}\left(a_{k}-a_{j}\right) \text {. }
$$

Then the evolution family $\{U(t, \tau)\}_{t \geq \tau}$ generated by (2.7), (2.3) is smoothing from $L^{2}(0,1)^{n}$ to $C^{1}([0,1])^{n}$.

Corollary 2.6 Assume that the unperturbed problem (2.1), (2.3) fulfills the conditions (2.4), (2.5), and (2.14). If $\tilde{b}_{j j} \equiv 0$ and (2.15) is fulfilled with $\tilde{b}_{j k}$ in place of $b_{j k}$, then the evolution family $\{\tilde{U}(t, \tau)\}_{t \geq \tau}$ generated by the perturbed problem (2.2), (2.3) is smoothing from $L^{2}(0,1)^{n}$ to $C^{1}([0,1])^{n}$.

The proof of the corollary straightforwardly follows from Theorem 2.5 and the fact that the condition (2.14) is stable with respect to perturbations $\tilde{b}$ of the matrix $b$ such that $\tilde{b}_{j j} \equiv 0$.

In Section 5.2 we prove the main result stating that the evolution family of the perturbed problem $(2.2),(2.3)$ is exponentially stable.

Theorem 2.7 Under the conditions (2.4), (2.5), (2.14) the following is true.

(८) For any $\gamma>0$ there exist $\varepsilon>0$ and $M=M(\gamma) \geq 1$ such that, whenever $\max _{j, k}\left\|\tilde{b}_{j k}\right\|_{\infty}<\varepsilon$, the evolution family $\{\tilde{U}(t, \tau)\}_{t \geq \tau}$ generated by the perturbed problem (2.2), (2.3) fulfills the bound

$$
\|\tilde{U}(t, \tau)\|_{\mathcal{L}\left(L^{2}(0,1)^{n}\right)} \leq M e^{-\gamma(t-\tau)} \quad \text { for } t \geq \tau
$$

(८) If $\tilde{b}_{j j} \equiv 0$ and (2.15) is fulfilled with $\tilde{b}_{j k}$ in place of $b_{j k}$, then for any $\gamma>0$ there exist $\varepsilon>0$ and $M_{1}=M_{1}(\gamma) \geq M$ such that, whenever $\max _{j, k}\left\|\tilde{b}_{j k}\right\|_{1}<\varepsilon$, the evolution family $\{\tilde{U}(t, \tau)\}_{t \geq \tau}$ fulfills the bound

$$
\|\tilde{U}(t, \tau)\|_{\mathcal{L}\left(L^{2}(0,1)^{n}, C^{1}([0,1])^{n}\right)} \leq M_{1} e^{-\gamma(t-\tau)} \text { for } t \geq \tau+T_{1}
$$

for some constant $T_{1}>0$.

Remark 2.8 The assumption $\tilde{b}_{j j} \equiv 0$ of Theorem 2.7 ( $\iota$ ) can be dropped in some cases (see Examples 3.4 and 3.5). However, we cannot avoid it in general (see Example 3.7). The reason is that the condition (2.14), ensuring the smoothing property for the perturbed problem, can be destroyed by perturbations $\tilde{b}_{j j}$ of $b_{j j}$.

\section{Examples and comments}

\subsection{Condition (2.15) is essential}

The condition (2.15) is a kind of Levy condition for compensating weak hyperbolicity. Let us show that it is crucial for the regularity result stated in Theorem 2.5. 
Example 3.1 Let $\varphi: \mathbb{R} \rightarrow \mathbb{R}$ be a continuous 1-periodic function which is continuously differentiable on $(0,1)$. Consider the following problem in $\Pi_{0}$ :

$$
\begin{gathered}
\partial_{t} u_{1}+\partial_{x} u_{1}=0, \\
\partial_{t} u_{2}+\partial_{x} u_{2}-u_{1}=0, \\
u_{1}(x, 0)=\varphi(x), \\
u_{2}(x, 0)=x \varphi(x), \\
u_{1}(0, t)=u_{2}(1, t), \\
u_{2}(0, t)=0
\end{gathered}
$$

This problem is a particular case of (2.7), (2.6), (2.3) and satisfies all assumptions of Theorem 2.5 except (2.15). It is straightforward to check that

$$
u_{1}=\varphi(x-t), \quad u_{2}=x \varphi(x-t)
$$

is a continuous solution to the problem (3.1), (3.2), (3.3). One can easily see that this solution is not continuously differentiable even if $t$ is supposed to be large, since its regularity does not exceed the regularity of the initial function $\varphi(x)$ for any $t$. Thus, the conclusion of Theorem 2.5 cannot be ensured without (2.15).

\subsection{Theorem 2.7 is not true under "large" perturbations}

Here we present simple examples showing that if the entries of the perturbation matrix $\tilde{b}$ are not small enough, then the statement of Theorem 2.7 about the exponential stability of (2.2), (2.6), (2.3) fails.

Example 3.2 In $\Pi_{0}$, let us consider the $2 \times 2$-system

$$
\begin{aligned}
& \partial_{t} u_{1}+\partial_{x} u_{1}=\nu u_{2} \\
& \partial_{t} u_{2}-\partial_{x} u_{2}=0
\end{aligned}
$$

with the boundary conditions

$$
u_{1}(0, t)=0, \quad u_{2}(1, t)=u_{1}(1, t)
$$

Note that the unperturbed problem (when $\nu=0$ ) is superstable. We now show that, if $\nu>1$, then (3.4), (3.5) is not exponentially stable. To this end, consider the corresponding spectral problem

$$
\begin{aligned}
& \lambda v_{1}+v_{1}^{\prime}=\nu v_{2}, \quad \lambda v_{2}-v_{2}^{\prime}=0, \quad 0<x<1, \\
& v_{1}(0)=0, \quad v_{2}(1)=v_{1}(1),
\end{aligned}
$$

with spectral parameter $\lambda$. All solutions are given by the formulas

$$
v_{1}(x, \lambda)=C e^{-\lambda x}+C_{1} \frac{\nu}{2 \lambda} e^{\lambda x}, \quad v_{2}(x, \lambda)=C_{1} e^{\lambda x} .
$$


The boundary conditions imply that the problem (3.6) has a nonzero solution if and only if the spectral parameter $\lambda$ satisfies the characteristic equation

$$
\frac{\nu}{2 \lambda}\left(e^{\lambda}-e^{-\lambda}\right)=e^{\lambda}, \quad \lambda \neq 0
$$

Setting $\gamma=2 \lambda$, we get

$$
\nu\left(1-e^{-\gamma}\right)=\gamma
$$

If $\nu>1$, then this equation has a positive solution $\gamma$ and, hence the spectral problem (3.6) has a positive eigenvalue. Due to the spectral mapping theorem, the problem (3.4), (3.5) for $\nu>1$ is not exponentially stable.

In [9] it is proved that, if $|\nu|<1$, then all non-zero solutions to (3.7) have negative real parts uniformly separated from zero. This means that the problem (3.4), (3.5) is exponentially stable for $|\nu|<1$.

Example 3.3 Now in $\Pi_{0}$ we consider a $2 \times 2$-system with a diagonal lower-order part, namely

$$
\begin{aligned}
& \partial_{t} u_{1}+\partial_{x} u_{1}+\mu u_{1}-\nu u_{2}=0, \partial_{t} u_{2}-\partial_{x} u_{2}=0, \quad x \in(0,1) \\
& u_{1}(0, t)=0, \quad u_{1}(1, t)=u_{2}(1, t),
\end{aligned}
$$

where $\mu$ and $\nu$ are positive constants. The unperturbed problem $(\nu=0)$ is superstable. Our aim is to show that the smallness of $\nu$ required to ensure the exponential stability of the problem (3.8) can be expressed in terms of the diagonal lower-order coefficient $\mu$.

The corresponding eigenvalue problem reads

$$
\begin{aligned}
& v_{1}^{\prime}+(\mu+\lambda) v_{1}-\nu v_{2}=0, v_{2}^{\prime}-\lambda v_{2}=0, \quad x \in(0,1) \\
& v_{1}(0)=0, v_{1}(1)-v_{2}(1)=0
\end{aligned}
$$

where $\lambda$ is a spectral parameter. The system (3.9) is equivalent to

$$
v_{1}(x)=\frac{c \nu}{2 \lambda+\mu}\left(e^{\lambda x}-e^{-(\mu+\lambda) x}\right), v_{2}(x)=c e^{\lambda x},
$$

where

$$
\nu e^{-\mu-2 \lambda}=\nu-2 \lambda-\mu \text {. }
$$

Here $c=v_{2}(0)$ is a nonzero complex constant. Setting $z=2 \lambda$, we come to the characteristic quasipolynomial equation for $z$, namely

$$
z+a+b e^{-z}=0
$$

where $a=\mu-\nu, b=\nu e^{-\mu}$. This equation is in detail analyzed in [9] in the context of asymptotic stability for ordinary differential equations with retarded arguments. Accordingly to the results obtained in [9], if

$$
\nu<\frac{\mu}{1+e^{-\mu}}
$$


then the solutions to (3.10) have negative real parts, uniformly separated from zero. Hence, the problem (3.8) is exponentially stable. Moreover, if

$$
\nu>\frac{\mu+1}{1-e^{-\mu}}
$$

then at least one solution to (3.10) has a positive real part, which implies that the problem (3.8) for such $\mu$ and $\nu$ is not exponentially stable.

Note that the condition of smallness similar to (3.11) will naturally appear in the proof of Theorem 2.7; cf. the equality (5.12).

\subsection{Condition (2.14) appears in applications}

It is worth to note that the condition (2.14) is fulfilled in many applications.

Example 3.4 The papers [34, 35] discuss catalytic processes in a chemical reactor. A chemical reaction is of zero order if the reaction rate does not depend on the amount of reactants. Such reactions are described by the following boundary value problem for a $2 \times 2$-semilinear hyperbolic system in $\Pi_{0}$ :

$$
\begin{aligned}
\beta \partial_{t} \Theta+\partial_{x} \Theta & =Q K e^{\Theta}-\mu\left(\Theta-\Theta_{r}\right), \\
\partial_{t} \Theta_{r}-\partial_{x} \Theta_{r} & =\mu\left(\Theta-\Theta_{r}\right) \\
\Theta(0, t) & =\Theta_{r}(0, t) \\
\Theta_{r}(1, t) & =\vartheta_{0}
\end{aligned}
$$

where $\Theta$ denotes the temperature in the reactor and $\Theta_{r}$ the temperature in the refrigerator. Moreover, $\beta, \mu, K, Q$, and $\vartheta_{0}$ are positive constants characterizing a catalyst and a reactant. It is supposed that the initial data $\Theta(x, 0)$ and $\Theta_{r}(x, 0)$ are given.

Linearization of the problem (3.13) at the stationary solution $\Theta_{0}(x), \Theta_{r 0}(x)$, whose existence is proved in $[30,34]$, is a boundary value problem with respect to $u=\Theta-\Theta_{0}$ and $v=\Theta_{r}-\Theta_{r 0}$, namely the system

$$
\begin{aligned}
\beta \partial_{t} u+\partial_{x} u & =\left(Q K e^{\Theta_{0}}-\mu\right) u+\mu v \\
\partial_{t} v-\partial_{x} v & =\mu(u-v)
\end{aligned}
$$

subjected to the boundary conditions

$$
u(0, t)=v(0, t), \quad v(1, t)=0
$$

and the initial conditions $u(x, 0)=u_{0}(x), v(x, 0)=v_{0}(x)$.

Note that the system (3.14), (3.15) is a perturbation of the following superstable system:

$$
\begin{aligned}
\beta \partial_{t} u+\partial_{x} u & =Q K e^{\Theta_{0}} u, \\
\partial_{t} v-\partial_{x} v & =0 \\
u(0, t) & =v(0, t), \\
v(1, t) & =0 .
\end{aligned}
$$


The condition (2.14) is here fulfilled with $k=2$, that is, $(C P)^{2} z=0$ for all $z=(u, v) \in$ $C(\bar{\Pi})^{2}$. Indeed,

$$
\begin{aligned}
(C P z)_{1}(x, t) & =e^{\int_{0}^{x} b_{11}(\eta) d \eta} v(0, t-\beta x), \\
(C P z)_{2}(x, t) & =0 \\
\left((C P)^{2} z\right)_{1}(x, t) & =e^{\int_{0}^{x} b_{11}(\eta) d \eta+\int_{0}^{1} b_{22}(\eta) d \eta} v(1, t-\beta x-1)=0,
\end{aligned}
$$

where $b_{11}(x)=Q K e^{\Theta_{0}(x)}$ and $b_{22}(x)=0$.

From Theorem $2.7(\iota)$ it follows that, for sufficiently small $\mu$, the evolution family $U(t, \tau)$ generated by the linear problem $(3.14),(3.15)$ is uniformly exponentially stable in $L^{2}$, with an exponential decay rate $\gamma>0$. This means that the eigenvalues of the corresponding eigenvalue problem have negative real parts uniformly separated from zero. By the linearization principle for autonomous non-linear strictly hyperbolic systems in $C^{1}$, proved in [10], the corresponding $C^{1}$-stationary solution to the nonlinear problem (3.13) is exponentially stable in $C^{1}$, with an exponential decay rate $\gamma^{\prime}$ such that $0<\gamma^{\prime}<\gamma$.

On the other side, from the proof of Theorem $2.7(\iota)$ one can easily see the following: For sufficiently small $\mu$, the problem (3.14), (3.15) is uniformly exponentially stable not only in $L^{2}$ but also in $C^{1}$, despite $\tilde{b}_{11}=\tilde{b}_{22}=\mu \neq 0$ (while in Theorem 2.7 ( $\iota$ ) we have $\left.\tilde{b}_{11}=\tilde{b}_{22} \equiv 0\right)$. Our argument works out because the condition (2.14), which entails the smoothing property of the perturbed problem, is stable with respect to perturbations of $b_{j j}$, which entails the smoothing property of the perturbed operator (in general this is not true, see Example 3.7 below). Indeed, the condition (2.14) for the perturbed problem (3.14), (3.15) is fulfilled with $k=2$, and the equalities (3.16) are true with $b_{11}(x)=Q K e^{\Theta_{0}(x)}-\mu$ and $b_{22}(x)=-\mu$.

Example 3.5 A chemical reaction is of first order if the reaction rate depends linearly on the concentration of reactants. In the presence of a catalyst and the internal heat exchange, first order reactions are described by the following initial-boundary value problem in $\Pi_{0}$ for a $3 \times 3$-semilinear hyperbolic system:

$$
\begin{aligned}
\beta \partial_{t} \Theta+\partial_{x} \Theta & =Q K e^{\Theta}(1-C)-\mu\left(\Theta-\Theta_{r}\right), \\
\partial_{t} C+\partial_{x} C & =K(1-C) e^{\Theta}, \\
\partial_{t} \Theta_{r}-\partial_{x} \Theta_{r} & =\mu\left(\Theta-\Theta_{r}\right), \\
\Theta(0, t) & =\Theta_{r}(0, t), \\
C(0, t) & =0 \\
\Theta_{r}(1, t) & =\vartheta_{0},
\end{aligned}
$$

where $C$ is the concentration of the reactant. The initial data $\Theta(x, 0), C(x, 0)$, and $\Theta_{r}(x, 0)$ are supposed to be given.

In $[30,34]$ it is proved that the problem has a stationary solution for certain parameters. Note that the linearization of (3.17) in a neighborhood of the stationary solution $\Theta_{0}(x), C_{0}(x), \Theta_{r 0}(x)$ is a particular case of our problem. Indeed, with respect to 
$u=\Theta-\Theta_{0}, v=C-C_{0}$, and $w=\Theta_{r}-\Theta_{r 0}$, it is represented by the system

$$
\begin{aligned}
\beta \partial_{t} u+\partial_{x} u & =\left(Q K e^{\Theta_{0}}\left(1-C_{0}\right)-\mu\right) u-Q K e^{\Theta_{0}} v+\mu w \\
\partial_{t} v+\partial_{x} v & =K e^{\Theta_{0}}\left(1-C_{0}\right) u-K e^{\Theta_{0}} v \\
\partial_{t} w-\partial_{x} w & =\mu(u-w)
\end{aligned}
$$

with the boundary conditions

$$
u(0, t)=w(0, t), \quad v(0, t)=0, \quad w(1, t)=0
$$

and the initial conditions $u(x, 0)=u_{0}(x), v(x, 0)=v_{0}(x), w(x, 0)=w_{0}(x)$.

Note that the system (3.18), (3.19) is a perturbation of a superstable system, namely

$$
\begin{aligned}
\beta \partial_{t} u+\partial_{x} u & =0 \\
\partial_{t} v+\partial_{x} v & =0 \\
\partial_{t} w-\partial_{x} w & =0 \\
u(0, t) & =w(0, t), \\
v(0, t) & =0 \\
w(1, t) & =0 .
\end{aligned}
$$

Again, the condition (2.14) is fulfilled here with $k=2$. Indeed,

$$
\begin{aligned}
(C P z)_{1}(x, t) & =e^{\int_{0}^{x} b_{11}(\eta) d \eta} w(0, t-\beta x), \\
(C P z)_{2}(x, t) & =(C P z)_{3}(x, t)=0, \\
\left((C P)^{2} z\right)_{1}(x, t) & =e^{\int_{0}^{x} b_{11}(\eta) d \eta+\int_{0}^{1} b_{33}(\eta) d \eta} w(1, t-\beta x-1)=0,
\end{aligned}
$$

where $z=(u, v, w)$ and $b_{11}(x)=b_{33}(x) \equiv 0$.

Similarly to the previous example, the linear problem (3.18), (3.19) is uniformly exponentially stable in $L^{2}$ for all sufficiently small $\mu$ and $K$. Moreover, the corresponding $C^{1}$-stationary solution to the nonlinear problem (3.17) is exponentially stable in $C^{1}$.

The condition (2.14) is, again, stable with respect to perturbations of $b_{j j}$. This follows from the formulas (3.20) where for the perturbed problem (3.18), (3.19) we have $b_{11}(x)=$ $Q K e^{\Theta_{0}(x)}\left(1-C_{0}(x)\right)-\mu$ and $b_{33}(x)=-\mu$. Using now the same argument as in Example 3.4, we conclude that for all sufficiently small $\mu$ and $K$ the problem (3.18), (3.19) is uniformly exponentially stable also in $C^{1}$.

Example 3.6 Another example is given by the following nonlinear problem in $\Pi_{0}$ that comes from the boundary control theory [25]:

$$
\begin{aligned}
\partial_{t} u+a_{1} \partial_{x} u & =b(x) u+c(x) v+u v, \\
\partial_{t} v-a_{2} \partial_{x} v & =(1-b(x)) u+(1-c(x)) v-u v, \\
u(0, t) & =0 \\
v(1, t) & =h\left(u(1, t), \lambda, u_{d}\right),
\end{aligned}
$$

where $a_{1}>0, a_{2}>0, \lambda$ is a control parameter based on the boundary measurement $u(1, t)$, and $u_{d}$ denotes the desired level required for the signal output $u(1, t)$. Again, the linearization is covered by (2.7), (2.3) and fulfills (2.14) with $k=2$. 


\subsection{Our results apply to nonlinear problems}

Note that in Examples 3.4-3.6 above we deal with nonlinear problems.

\subsection{Condition (2.14) is in general not stable with respect to perturbations of $b_{j j}$}

Example 3.7 Let us consider the superstable system perturbed in the diagonal lower order part, namely

$$
\partial_{t} u_{1}+\partial_{x} u_{1}=0, \quad \partial_{t} u_{2}+\partial_{x} u_{2}=\varepsilon u_{2}, \quad \partial_{t} u_{3}-\partial_{x} u_{3}=0,
$$

and supplement it with the reflection boundary conditions

$$
u_{1}(0, t)=u_{3}(0, t), \quad u_{2}(0, t)=u_{3}(0, t), \quad u_{3}(1, t)=u_{1}(1, t)-u_{2}(1, t) .
$$

One can easily check that for the unperturbed problem $(\varepsilon=0)$ the condition (2.14) is fulfilled with $k=3$, while for the perturbed problem $(\varepsilon \neq 0)$ it is not fulfilled for any $k \in \mathbb{N}$. This follows from the following simple calculations:

$$
\begin{aligned}
(C P z)_{1}(x, t) & =u_{3}(0, t-x), \\
(C P z)_{2}(x, t) & =e^{\varepsilon x}(C P z)_{1}(x, t), \\
(C P z)_{3}(x, t) & =u_{1}(1, t+x-1)-u_{2}(1, t+x-1), \\
\left((C P)^{2} z\right)_{1}(x, t) & =u_{1}(1, t-x-1)-u_{2}(1, t-x-1), \\
\left((C P)^{2} z\right)_{2}(x, t) & =e^{\varepsilon x}\left((C P)^{2} z\right)_{1}(x, t), \\
\left((C P)^{2} z\right)_{3}(x, t) & =\left(1-e^{\varepsilon}\right) u_{3}(0, t+x-2), \\
\left((C P)^{3} z\right)_{1}(x, t) & =\left(1-e^{\varepsilon}\right) u_{3}(0, t-x-2), \\
\left((C P)^{3} z\right)_{2}(x, t) & =e^{\varepsilon x}\left((C P)^{3} z\right)_{1}(x, t), \\
\left((C P)^{3} z\right)_{3}(x, t) & =\left(1-e^{\varepsilon}\right)\left(u_{1}(1, t+x-3)-u_{2}(1, t+x-3)\right),
\end{aligned}
$$

where $z=\left(u_{1}, u_{2}, u_{3}\right)$.

\section{Evolution families}

In this section for the problem $(2.7),(2.6),(2.3)$ we prove the existence of an evolution family on $L^{2}(0,1)^{n}$ and show that it is eventually differentiable. Existence of evolution families on the spaces of continuous functions is a complicated question because one has to take into account compatibility conditions between initial and boundary data. The point is that the first order compatibility conditions depend on the coefficients of the differential equations and are not the same for unperturbed and perturbed problems. This complication can be avoided (and we will follow this way) working with evolution operators defined on $L^{2}(0,1)^{n}$, where the compatibility conditions do not play any role. Nevertheless, the main result (Theorem 2.7 stating the exponential stability) will be proved in both $L^{2}(0,1)^{n}$ and $C^{1}([0,1])^{n}$-spaces. For the latter we will use the result in $L^{2}(0,1)^{n}$ and the smoothing property of the evolution families in the sense of Definition 2.4 . 


\subsection{A priori estimates}

The following results about existence and uniqueness of continuous and classical solutions to the problem $(2.7),(2.6),(2.3)$ readily follow from $[15,18]$.

Theorem 4.1 [15, 18] Suppose that the coefficients $a_{j}$ and $b_{j j}$ fulfill the conditions (2.4), (2.5), and (2.8). Let $\tau \in \mathbb{R}$ be arbitrary fixed. If $\varphi \in C^{1}([0,1])^{n}$ fulfills the zero order compatibility conditions

$$
\begin{aligned}
\varphi_{j}(0) & =\sum_{k=1}^{m} p_{j k} \varphi_{k}(1)+\sum_{k=m+1}^{n} p_{j k} \varphi_{k}(0), \quad 1 \leq j \leq m, \\
\varphi_{j}(1) & =\sum_{k=1}^{m} p_{j k} \varphi_{k}(1)+\sum_{k=m+1}^{n} p_{j k} \varphi_{k}(0), \quad m<j \leq n,
\end{aligned}
$$

and the first order compatibility conditions

$$
\begin{aligned}
& \psi_{j}(0)=\sum_{k=1}^{m} p_{j k} \psi_{k}(1)+\sum_{k=m+1}^{n} p_{j k} \psi_{k}(0), \quad 1 \leq j \leq m, \\
& \psi_{j}(1)=\sum_{k=1}^{m} p_{j k} \psi_{k}(1)+\sum_{k=m+1}^{n} p_{j k} \psi_{k}(0), \quad m<j \leq n,
\end{aligned}
$$

where

$$
\psi(x)=-\left(a(x, \tau) \partial_{x}+b(x, \tau)\right) \varphi(x),
$$

then in $\Pi_{\tau}$ there exists a unique classical (continuously differentiable) solution $u(x, t)$ to the problem (2.7), (2.6), (2.3). Moreover, there are constants $K_{1} \geq 1$ and $\omega_{1}>0$ not depending on $\tau, t$, and $\varphi$ such that

$$
\|u(\cdot, t)\|_{C^{1}([0,1])^{n}} \leq K_{1} e^{\omega_{1}(t-\tau)}\|\varphi\|_{C^{1}([0,1])^{n}} \text { for } t \geq \tau .
$$

Given $c>0$, the constants $K_{1}$ and $\omega_{1}$ can be chosen the same for all $b_{j k}$ such that $\max _{j, k}\left\|b_{j k}\right\|_{1}<c$.

Lemma 4.2 If $u(x, t)$ is a classical solution to the problem (2.7), (2.6), (2.3), then it fulfills the estimate

$$
\|u(\cdot, t)\|_{L^{2}(0,1)^{n}} \leq K_{2} e^{\omega_{2}(t-\tau)}\|\varphi\|_{L^{2}(0,1)^{n}} \text { for } t \geq \tau
$$

with some constants $K_{2} \geq 1$ and $\omega_{2}>0$ not depending on $\tau$, $t$, and $\varphi$.

Proof. The proof is based on the argument from [11] used to get a priori estimates for initial-boundary value problems for first order hyperbolic systems, now for decoupled boundary conditions. Let $u=u(x, t)$ be a classical solution to the problem under consideration. Take a scalar product of (2.7) with $u$ and integrate the resulting system over the rectangle $\Pi_{\tau}^{t}=\{(x, \theta): 0<x<1, \tau<\theta<t\}$. We get

$$
\iint_{\Pi_{\tau}^{t}}\left(\frac{\partial}{\partial \theta}(u, u)+\frac{\partial}{\partial x}(a u, u)\right) d x d \theta=\iint_{\Pi_{\tau}^{t}}\left(-2(b u, u)+\left(a_{x} u, u\right)\right) d x d \theta
$$


where $(\cdot, \cdot)$ denotes the scalar product in $\mathbb{R}^{n}$. Applying Green's formula to the left hand side, we obtain

$$
\begin{gathered}
\|u(\cdot, t)\|_{L^{2}(0,1)^{n}}^{2}+\int_{\tau}^{t}\left(\sum_{j=1}^{n} a_{j}(1, \theta) u_{j}^{2}(1, \theta)-\sum_{j=1}^{n} a_{j}(0, \theta) u_{j}^{2}(0, \theta)\right) d \theta \\
=\|\varphi\|_{L^{2}(0,1)^{n}}^{2}+\iint_{\Pi_{\tau}^{t}}\left(-2(b u, u)+\left(a_{x} u, u\right)\right) d x d \theta
\end{gathered}
$$

Suppose first that the boundary conditions (2.3) are dissipative, i.e.

$\sum_{j=1}^{m} a_{j}(1, t) u_{j}^{2}(1, t)-\sum_{j=m+1}^{n} a_{j}(0, t) u_{j}^{2}(0, t)+\sum_{j=m+1}^{n} a_{j}(1, t)(P u)_{j}(t)^{2}-\sum_{j=1}^{m} a_{j}(0, t)(P u)_{j}(t)^{2} \geq 0$.

Then from (4.5) we have

$$
\begin{aligned}
\|u(\cdot, t)\|_{L^{2}(0,1)^{n}}^{2} & \leq\|\varphi\|_{L^{2}(0,1)^{n}}^{2}+\iint_{\Pi_{\tau}^{t}}\left|\left(\left(a_{x}-2 b\right) u, u\right)\right| d x d \theta \\
& \leq\|\varphi\|_{L^{2}(0,1)^{n}}^{2}+\beta \int_{\tau}^{t} I(\theta) d \theta
\end{aligned}
$$

where $I(t)=\|u(\cdot, t)\|_{L^{2}(0,1)^{n}}^{2}=\int_{0}^{1}(u, u) d x, \beta=n \max _{i, j}\left\|\left(a_{x}-2 b\right)_{i j}\right\|_{\infty}$, and $\left(a_{x}-2 b\right)_{i j} \in$ $B C(\Pi)$ are entries of the matrix $a_{x}-2 b$. Applying Gronwall's argument to the inequality

$$
I(t) \leq\|\varphi\|_{L^{2}(0,1)^{n}}^{2}+\beta \int_{\tau}^{t} I(\theta) d \theta
$$

we come to the estimate $\|u(\cdot, t)\|_{L^{2}(0,1)^{n}} \leq e^{\omega_{2}(t-\tau)}\|\varphi\|_{L^{2}(0,1)^{n}}$ with constant $\omega_{2}=\frac{\beta}{2}$ depending on the coefficients of the system (2.7), (2.3) but not on $\varphi$.

To complete the proof, it remains to show that the inequality (4.6), supposed above, causes no loss of generality. Let $\mu_{i}(x, t)$ be arbitrary smooth functions satisfying the conditions

$$
\inf _{\bar{\Pi}_{\tau}}\left|\mu_{j}\right|>0, \quad \sup _{\bar{\Pi}_{\tau}}\left|\mu_{j}\right|<\infty \quad \text { for all } j \leq n .
$$

The change of each variable $u_{j}$ to $v_{j}=\mu_{j} u_{j}$ brings the system (2.7) to

$$
\partial_{t} v_{j}+a_{j}(x, t) \partial_{x} v_{j}-\frac{\partial_{t} \mu_{j}+a_{j}(x, t) \partial_{x} \mu_{j}}{\mu_{j}} v_{j}+\sum_{k=1}^{n} b_{j k} \frac{\mu_{j}}{\mu_{k}} v_{k}=0
$$

and the boundary conditions (2.3) to

$$
\begin{array}{ll}
v_{j}(0, t)=\sum_{k=1}^{m} p_{j k} \frac{\mu_{j}(0, t)}{\mu_{k}(1, t)} v_{k}(1, t)+\sum_{k=m+1}^{n} p_{j k} \frac{\mu_{j}(0, t)}{\mu_{k}(0, t)} v_{k}(0, t), \quad 1 \leq j \leq m, \\
v_{j}(1, t)=\sum_{k=1}^{m} p_{j k} \frac{\mu_{j}(1, t)}{\mu_{k}(1, t)} v_{k}(1, t)+\sum_{k=m+1}^{n} p_{j k} \frac{\mu_{j}(1, t)}{\mu_{k}(0, t)} v_{k}(0, t), \quad m<j \leq n .
\end{array}
$$


Note that the resulting system (4.7), (4.8) is of the type (2.7), (2.3), and the inequality (4.6) for it reads

$$
\begin{aligned}
& \sum_{j=1}^{m} a_{j}(1, t) v_{j}^{2}(1, t)-\sum_{j=m+1}^{n} a_{j}(0, t) v_{j}^{2}(0, t) \\
& +\sum_{j=m+1}^{n} a_{j}(1, t)\left[\sum_{k=m+1}^{n} p_{j k} \frac{\mu_{j}(1, t)}{\mu_{k}(0, t)} v_{k}(0, t)+\sum_{k=1}^{m} p_{j k} \frac{\mu_{j}(1, t)}{\mu_{k}(1, t)} v_{k}(1, t)\right]^{2} \\
& -\sum_{j=1}^{m} a_{j}(0, t)\left[\sum_{k=m+1}^{n} p_{j k} \frac{\mu_{j}(0, t)}{\mu_{k}(0, t)} v_{k}(0, t)+\sum_{k=1}^{m} p_{j k} \frac{\mu_{j}(0, t)}{\mu_{k}(1, t)} v_{k}(1, t)\right]^{2} \geq 0 .
\end{aligned}
$$

One can easily see that the functions $\mu_{j}$ can be chosen so that the left hand side of (4.9) is a non-negative definite quadratic form with respect to $v_{j}(1, t), j \leq m$ and $v_{j}(0, t)$, $m+1 \leq j \leq n$. Indeed, since $a_{j}>0$ for $j \leq m$ and $a_{j}<0$ for $m+1 \leq j \leq n$ by the assumption (2.5), the first line of (4.9) is a positive definite quadratic form and the last two lines are a non-negative definite quadratic form. Now we choose the functions $\mu_{j}$ so that $\mu_{j}(0, t)$ for $m+1 \leq j \leq n$ and $\mu_{j}(1, t)$ for $j \leq m$ are so large, while $\mu_{j}(1, t)$ for $m+1 \leq j \leq n$ and $\mu_{j}(0, t)$ for $j \leq m$ are so small that the whole expression in (4.9) is a non-negative definite quadratic form.

This completes the proof.

\subsection{Existence of evolution families (proof of Theorem 2.3)}

Theorem 4.1 guarantees the existence and uniqueness of the classical solution to the problem (2.7), (2.6), (2.3) for any $\tau \in \mathbb{R}$ and any initial function $\varphi \in C_{0}^{\infty}([0,1])^{n}$. Fix arbitrary $\tau \in \mathbb{R}, \varphi \in L^{2}(0,1)^{n}$, and a sequence $\varphi^{l} \in C_{0}^{\infty}([0,1])^{n}$ such that $\varphi^{l} \rightarrow \varphi$ in $L^{2}(0,1)^{n}$. Let $u^{l}$ denote the continuously differentiable solutions to the problem (2.7), (2.6), (2.3) with $\varphi(x)=\varphi^{l}(x)$. The bound (4.4) implies that

$$
\max _{\tau \leq \theta \leq t}\left\|u^{m}(\cdot, \theta)-u^{l}(\cdot, \theta)\right\|_{L^{2}(0,1)^{n}} \rightarrow 0 \text { as } m, l \rightarrow \infty
$$

for each $t>\tau$. It follows that, given $t>\tau$, the sequence $u^{l}$ converges in $C\left([\tau, t], L^{2}(0,1)\right)^{n}$. Moreover, (4.4) ensures that the limit function $u$ does not depend on the choice of $\varphi^{l}$.

Define $U(t, \tau) \varphi=u(\cdot, t)$. Thus, $U(t, \tau) \varphi \in L^{2}(0,1)^{n}$ for each $t \geq \tau$ and $\varphi \in L^{2}(0,1)^{n}$. Since the classical solution to (2.7), (2.6), (2.3) is unique, the family $\{U(t, \tau)\}_{t \geq \tau}$ fulfills the first property in Definition 2.1. Moreover, the estimate (4.4) entails the exponential bound

$$
\|U(t, \tau)\|_{\mathcal{L}\left(L^{2}([0,1])^{n}\right)} \leq K_{2} e^{\omega_{2}(t-\tau)}, \quad t \geq \tau .
$$

as well as the second property in Definition 2.1. More specifically, the strong continuity of $U(t, \tau)$ in $t$ (and similarly in $\tau$ ) follows from the convergence

$$
\|U(t, \tau) \varphi-U(\tilde{t}, \tau) \varphi\|_{L^{2}(0,1)^{n}}=\|(U(t, \tilde{t})-I) U(\tilde{t}, \tau) \varphi\|_{L^{2}(0,1)^{n}} \rightarrow 0 \text { as } t \rightarrow \tilde{t} \quad(t \geq \tilde{t} \geq \tau)
$$


for all $\varphi \in L^{2}(0,1)^{n}$. To prove this convergence, it is sufficient to note that $U(\tilde{t}, \tau) \varphi \in$ $L^{2}(0,1)^{n}$ and to show that

$$
\|U(t, \tau) \varphi-\varphi\|_{L^{2}(0,1)^{n}} \rightarrow 0 \text { as } t \rightarrow \tau \quad(t \geq \tau)
$$

for all $\varphi \in L^{2}(0,1)^{n}$. To this end, given $\varphi \in L^{2}(0,1)^{n}$, take an arbitrary sequence $\varphi^{l} \in C_{0}^{\infty}([0,1])^{n}$ such that $\varphi^{l} \rightarrow \varphi$ in $L^{2}(0,1)^{n}$ as $l \rightarrow \infty$. Then

$$
\begin{aligned}
& \|U(t, \tau) \varphi-\varphi\|_{L^{2}(0,1)^{n}} \\
& \quad \leq\left\|U(t, \tau) \varphi-U(t, \tau) \varphi^{l}\right\|_{L^{2}(0,1)^{n}}+\left\|U(t, \tau) \varphi^{l}-\varphi^{l}\right\|_{L^{2}(0,1)^{n}}+\left\|\varphi^{l}-\varphi\right\|_{L^{2}(0,1)^{n}} .
\end{aligned}
$$

As $l \rightarrow \infty$, the first summand in the right-hand side tends to zero by (4.4). The second summand tends to zero because $U(t, \tau) \varphi^{l}$ for each $l$ is the classical solution. The third one tends to zero by the choice of $\varphi^{l}$. This completes the proof of (4.10) and, therefore, the strong continuity of $U(t, \tau)$ in $t$, as desired.

Therefore, $\{U(t, \tau)\}_{t \geq \tau}$ determines an exponentially bounded evolution family generated by the problem (2.7), (2.6) in the sense of Definitions 2.1 and 2.2. Theorem 2.3 is therewith proved.

This proof motivates the following definition of an $L^{2}$-generalized solution to the problem $(2.7),(2.6),(2.3)$.

Definition 4.3 Given $\varphi \in L^{2}(0,1)^{n}$, let $\varphi^{l} \in C_{0}^{\infty}([0,1])^{n}$ be an arbitrary fixed sequence such that $\varphi^{l} \rightarrow \varphi$ in $L^{2}(0,1)^{n}$. A function $u \in C\left([\tau, \infty), L^{2}(0,1)\right)^{n}$ is called an $L^{2}$ generalized solution to the problem (2.7), (2.6), (2.3) if the sequence of continuously differentiable solutions $u^{l}(x, t)$ to the problem (2.7), (2.6), (2.3) with $\varphi(x)=\varphi^{l}(x)$ fulfills the convergence

$$
\left\|u(\cdot, \theta)-u^{l}(\cdot, \theta)\right\|_{L^{2}(0,1)^{n}} \rightarrow_{l \rightarrow \infty} 0,
$$

uniformly in $\theta$ varying in the range $\tau \leq \theta \leq t$, for every $t>\tau$.

\subsection{Smoothing property}

In this section we show that, under the conditions (2.14) and (2.15), the $L^{2}$-generalized solutions to $(2.7),(2.6),(2.3)$ become continuous in a finite time. Furthermore, the time at which the solutions reach the $C$-regularity does not exceed the value $\tau+T_{0}$ for a fixed number $T_{0}>0$, whatsoever the initial time $\tau \in \mathbb{R}$ and the initial function $\varphi \in L^{2}(0,1)^{n}$. Furthermore, the function $u(x, t)=[U(t, \tau) \varphi](x)$ satisfies the zero order compatibility conditions at points $(0, t)$ and $(1, t)$. We summarize this in the following lemma. Let $Y_{0}$ denote the subspace of $C([0,1])^{n}$ of functions satisfying the zero-order compatibility conditions (4.1).

Lemma 4.4 Suppose that the conditions (2.4), (2.5), (2.8), (2.14), (2.15) are fulfilled. Then the evolution family $\{U(t, \tau)\}_{t \geq \tau}$ generated by the problem (2.7), (2.3) is smoothing from $L^{2}(0,1)^{n}$ to $Y_{0}$. 
The proof develops the ideas of $[16,17]$ where the smoothing property is proved from $Y_{0}$ to $C^{1}$, and it is shown that the solutions reach the $C^{k}$-regularity in a finite time for each $k$. Here we extend the smoothing results to the case where the initial data are $L^{2}$-functions only.

Proof. From Theorem 2.3 it follows that for all $\tau \in \mathbb{R}$ and $\varphi \in L^{2}(0,1)^{n}$ the problem (2.7), (2.6), (2.3) has a unique $L^{2}$-generalized solution $u$. Note that $u \in C\left([\tau, \infty), L^{2}(0,1)\right)^{n}$. It suffices to show that $u \in C\left(\bar{\Pi}_{\tau+T_{0}}\right)^{n}$ for some $T_{0}>0$ not depending on $\tau$ and $\varphi$.

Fix an arbitrary $\tau \in \mathbb{R}$ and $\varphi \in L^{2}(0,1)^{n}$. Due to (2.14), we can fix $d>0$ such that $\left((C P)^{k} u\right)(x, t)=0$ for all $(x, t) \in \bar{\Pi}_{\tau+d}$. Fix an arbitrary sequence of functions $\varphi^{l} \in C_{0}^{\infty}([0,1])^{n}, l \in \mathbb{N}$, such that $\varphi^{l} \rightarrow \varphi$ in $L^{2}(0,1)^{n}$. Let $u^{l}$ denote the classical solutions to the problem (2.7), (2.6), (2.3) with $\varphi(x)=\varphi^{l}(x)$. Due to the bound (4.4), $u^{l} \rightarrow u$ in $C\left([\tau, \theta], L^{2}(0,1)\right)^{n}$ as $l \rightarrow \infty$ for any $\theta>\tau$. In order to prove the lemma, it is sufficient to show that

$$
u^{l} \text { converges in } C\left(\bar{\Pi}_{\tau+2 d}^{\tau+2 d+\alpha}\right)^{n} \text { as } l \rightarrow \infty,
$$

for any $\alpha>0$. Here and below, given $\beta$ and $\gamma$ such that $\beta<\gamma$, we use the notation $\Pi_{\beta}^{\gamma}=\Pi_{\beta} \backslash \bar{\Pi}_{\gamma}$.

Given $\alpha>0$, let a linear bounded operator $D: C\left(\bar{\Pi}_{\tau}^{\tau+2 d+\alpha}\right)^{n} \mapsto C\left(\bar{\Pi}_{\tau}^{\tau+2 d+\alpha}\right)^{n}$ be defined by the formula

$$
(D w)_{j}(x, t)=-\int_{x_{j}(x, t)}^{x} d_{j}(\xi, x, t) \sum_{k \neq j} b_{j k}\left(\xi, \omega_{j}(\xi)\right) w_{k}\left(\xi, \omega_{j}(\xi)\right) d \xi, \quad j \leq n,
$$

where the functions $\omega_{j}(\xi, x, t)$ are given by (2.9). For simplicity, here and in what follows we use the notation $\omega_{j}(\xi)=\omega_{j}(\xi, x, t)$. Moreover, we drop the dependence of $D$ on $\alpha$, as throughout the proof $\alpha$ is arbitrary fixed.

Accordingly to (2.12), the solution $u^{l} \in C^{1}\left(\bar{\Pi}_{\tau}\right)^{n}$ satisfies the operator equation

$$
u^{l}=Q u^{l}+D u^{l} .
$$

In particular,

$$
\left.u^{l}\right|_{\bar{\Pi}_{\tau+d}}=C P u^{l}+D u^{l} .
$$

Putting (4.13) into the first summand in (4.13), we get

$$
\left.u^{l}\right|_{\bar{\Pi}_{\tau+d}}=(C P)^{2} u^{l}+(I+C P) D u^{l} .
$$

Iterating this, that is, substituting (4.13) into the last equation once and once again, in the $k$-th step we meet the property (2.14) and get the formula

$$
\left.u^{l}\right|_{\bar{\Pi}_{\tau+d}}=\sum_{i=0}^{k-1}(C P)^{i} D u^{l} .
$$


Since $u^{l}$ occurs in both sides of (4.14), this equation can be iterated. Note that $D$ operates with $u^{l}$ on a different (shifted) domain. Hence, such iteration is possible only on a subdomain of $\bar{\Pi}_{\tau+d}$. This is possible on $\bar{\Pi}_{\tau+2 d}$ and, doing so, we obtain

$$
\left.u^{l}\right|_{\bar{\Pi}_{\tau+2 d}}=\sum_{i=0}^{k-1}(C P)^{i} D \sum_{j=0}^{k-1}(C P)^{j} D u^{l}=\sum_{i=0}^{k-1}(C P)^{i} D \sum_{j=1}^{k-1}(C P)^{j} D u^{l}+\sum_{i=0}^{k-1}(C P)^{i} D^{2} u^{l} .(4
$$

We now have to prove that the right-hand side of (4.15) converges in $C\left(\bar{\Pi}_{\tau+2 d}^{\tau+2 d+\alpha}\right)^{n}$ as $l \rightarrow \infty$ for all $\alpha>0$. Fix an arbitrary $\alpha>0$. It suffices to show that

$$
\text { the sequences } D(C P)^{i} D u^{l} \text { and } D^{2} u^{l} \text { converge in } C\left(\bar{\Pi}_{\tau+2 d}^{\tau+2 d+\alpha}\right)^{n}
$$

as $l \rightarrow \infty$ for all $i=0,1, \ldots, k-1$. The proof of (4.16) will be divided into two claims.

Claim 1. The sequence $D^{2} u^{l}$ converges in $C\left(\bar{\Pi}_{\tau+2 d}^{\tau+2 d+\alpha}\right)^{n}$ as $l \rightarrow \infty$. On the account of (4.11), after changing the order of integration, we derive the following formula for $\left[D^{2} u^{l}\right]_{j}(x, t)$ on $\bar{\Pi}_{\tau+2 d}^{\tau+2 d+\alpha}$ for each $j \leq n$ :

$$
\left[D^{2} u^{l}\right]_{j}(x, t)=\sum_{k \neq j}^{n} \sum_{i \neq k}^{n} \int_{x_{j}}^{x} \int_{\eta}^{x} d_{j k i}(\xi, \eta, x, t) b_{j k}\left(\xi, \omega_{j}(\xi)\right) u_{i}^{l}\left(\eta, \omega_{k}\left(\eta, \xi, \omega_{j}(\xi)\right)\right) d \xi d \eta,(4,1
$$

where $x_{j}$ is given by $(2.10)$ and

$$
d_{j k i}(\xi, \eta, x, t)=d_{j}(\xi, x, t) d_{k}\left(\eta, \xi, \omega_{j}(\xi)\right) b_{k i}\left(\eta, \omega_{k}\left(\eta, \xi, \omega_{j}(\xi)\right)\right) .
$$

Note that, due to (2.15), given $j \leq n$ and $k \neq j$, the function $b_{j k}$ vanishes for those $\xi \in[0,1]$ such that $a_{k}\left(\xi, \omega_{j}(\xi)\right)=a_{j}\left(\xi, \omega_{j}(\xi)\right)$.

Now, for fixed $k \neq j$ and $\eta$, let us change the variables

$$
\xi \mapsto \theta=\omega_{k}\left(\eta, \xi, \omega_{j}(\xi)\right) .
$$

Taking into account the equalities

$$
\begin{gathered}
\partial_{x} \omega_{j}(\xi, x, t)=-\frac{1}{a_{j}(x, t)} \exp \int_{\xi}^{x} \frac{\partial_{t} a_{j}\left(\eta, \omega_{j}(\eta)\right)}{a_{j}\left(\eta, \omega_{j}(\eta)\right)^{2}} d \eta, \\
\partial_{t} \omega_{j}(\xi, x, t)=\exp \int_{\xi}^{x} \frac{\partial_{t} a_{j}\left(\eta, \omega_{j}(\eta)\right)}{a_{j}\left(\eta, \omega_{j}(\eta)\right)^{2}} d \eta,
\end{gathered}
$$

from (4.18) we get

$$
\begin{aligned}
d \theta & =\left[\partial_{2} \omega_{k}\left(\eta, \xi, \omega_{j}(\xi)\right)+\partial_{3} \omega_{k}\left(\eta, \xi, \omega_{j}(\xi)\right) \partial_{\xi} \omega_{j}(\xi)\right] d \xi \\
& =\frac{a_{k}\left(\xi, \omega_{j}(\xi)\right)-a_{j}\left(\xi, \omega_{j}(\xi)\right)}{a_{j}\left(\xi, \omega_{j}(\xi)\right) a_{k}\left(\xi, \omega_{j}(\xi)\right)} \partial_{3} \omega_{k}\left(\eta, \xi, \omega_{j}(\xi)\right) d \xi,
\end{aligned}
$$

where $\partial_{i}$ here and in what follows denotes the partial derivative with respect to the $i$-th argument. It follows from (4.19) that (4.18) is non-degenerate for all $\xi \in[0,1]$ fulfilling 
the condition $a_{k}\left(\xi, \omega_{j}(\xi)\right) \neq a_{j}\left(\xi, \omega_{j}(\xi)\right)$. Hence, for given $\eta, \theta, x$, and $t$, there exists a unique solution $\xi=\tilde{x}(\theta, \eta, x, t)$ to the equation $\omega_{k}(\xi, \eta, \theta)=\omega_{j}(\xi, x, t)$, and we have

$$
\omega_{k}(\tilde{x}(\theta, \eta, x, t), \eta, \theta)=\omega_{j}(\tilde{x}(\theta, \eta, x, t), x, t) .
$$

Changing the variables according to (4.18), we obtain

$$
\begin{aligned}
\int_{x_{j}}^{x} & \int_{\eta}^{x} d_{j k i}(\xi, \eta, x, t) b_{j k}\left(\xi, \omega_{j}(\xi)\right) u_{i}^{l}\left(\eta, \omega_{k}\left(\eta, \xi, \omega_{j}(\xi)\right)\right) d \xi d \eta \\
& =\int_{x_{j}}^{x} \int_{\omega_{j}(\eta, x, t)}^{\omega_{k}(\eta, x, t)} d_{j k i}(\tilde{x}, \eta, x, t) \beta_{j k}\left(\tilde{x}, \omega_{j}(\tilde{x})\right) \frac{\left(a_{k} a_{j}\right)\left(\tilde{x}, \omega_{j}(\tilde{x})\right)}{\partial_{3} \omega_{k}\left(\eta, \tilde{x}, \omega_{j}(\tilde{x})\right)} u_{i}^{l}(\eta, \theta) d \theta d \eta
\end{aligned}
$$

where $\beta_{j k}$ are continuous functions fulfilling (2.15). Note that $\beta_{j k}(x, t)$ are not uniquely defined by (2.15) for $(x, t)$ such that $a_{j}(x, t)=a_{k}(x, t)$. Nevertheless, the left-hand side and, hence, the right-hand side of (4.20) do not depend on the choice of $\beta_{j k}$. This easily follows from (2.15) and (4.19), entailing that $b_{j k}\left(\xi, \omega_{j}(\xi)\right)=0$ and $d \theta=0$ if $a_{j}\left(\xi, \omega_{j}(\xi)\right)=$ $a_{k}\left(\xi, \omega_{k}(\xi)\right)$. Changing the order of integration in the right-hand side of (4.20), we rewrite it as follows (where for definiteness we suppose that $j, k \leq m$ and $a_{k}<a_{j}$, hence $\omega_{j}(\xi)<$ $\omega_{k}(\xi)$; the other cases are treated similarly):

$$
\begin{aligned}
\int_{\omega_{j}(0)}^{\omega_{k}(0)} & \int_{0}^{\tilde{\omega}_{j}(\theta)} d_{j k i}(\tilde{x}, \eta, x, t) \beta_{j k}\left(\tilde{x}, \omega_{j}(\tilde{x})\right) \frac{\left(a_{k} a_{j}\right)\left(\tilde{x}, \omega_{j}(\tilde{x})\right)}{\partial_{3} \omega_{k}\left(\eta, \tilde{x}, \omega_{j}(\tilde{x})\right)} u_{i}^{l}(\eta, \theta) d \eta d \theta \\
& +\int_{\omega_{k}(0)}^{t} \int_{\tilde{\omega}_{k}(\theta)}^{\tilde{\omega}_{j}(\theta)} d_{j k i}(\tilde{x}, \eta, x, t) \beta_{j k}\left(\tilde{x}, \omega_{j}(\tilde{x})\right) \frac{\left(a_{k} a_{j}\right)\left(\tilde{x}, \omega_{j}(\tilde{x})\right)}{\partial_{3} \omega_{k}\left(\eta, \tilde{x}, \omega_{j}(\tilde{x})\right)} u_{i}^{l}(\eta, \theta) d \eta d \theta
\end{aligned}
$$

where $\tilde{\omega}_{s}(\tau)=\tilde{\omega}_{s}(\tau, x, t)$ denotes the inverse of the function from $[0,1]$ to $\mathbb{R}$ taking $\xi$ to $\tau=\omega_{s}(\xi, x, t)$. Note that the range of integration in $\theta$ in both integrals does not exceed $d$ in length. This follows from the fact that the time needed to reach the boundary $x=0$ or $x=1$ from any point $(x, t) \in \bar{\Pi}$ is not larger than $d$. The $C\left(\bar{\Pi}_{\tau+2 d}^{\tau+2 d+\alpha}\right)^{n}$-norm of the function (4.21) can be estimated from above by

$$
\begin{gathered}
\left.2 d \max _{x, \xi, \eta \in[0,1]} \max _{t, \theta \in[\tau, \tau+2 d+\alpha]} \mid d_{j k i}(\xi, \eta, x, t)\right)\left.\beta_{j k}(\xi, \theta) \frac{\left(a_{k} a_{j}\right)(\xi, \theta)}{\partial_{3} \omega_{k}(\eta, \xi, \theta)}\right|_{t \in[\tau, \tau+2 d+\alpha]} \max _{0}^{1}\left|u_{i}^{l}(\eta, t)\right| d \eta \\
\leq K\left\|u_{i}^{l}\right\|_{C\left([\tau, \tau+2 d+\alpha], L^{2}(0,1)\right)}
\end{gathered}
$$

where $K>0$ is a constant that depends on the coefficients $a$ and $b$ but does not depend on the function $u^{l}$. Thus Claim 1 is proved.

Claim 2. The sequence DCPDu converges in $C\left(\bar{\Pi}_{\tau+2 d}^{\tau+2 d+\alpha}\right)^{n}$ as $l \rightarrow \infty$. We have

$$
\begin{aligned}
& {\left[D C P D u^{l}\right]_{j}(x, t)=} \\
& =\sum_{k \neq j}^{n} \int_{x}^{x_{j}} d_{j}(\xi, x, t) b_{j k}\left(\xi, \omega_{j}(\xi)\right) c_{k}\left(x_{k}, \xi, \omega_{j}(\xi)\right)\left(P D u^{l}\right)_{k}\left(\omega_{k}\left(x_{k}, \xi, \omega_{j}(\xi)\right)\right) d \xi
\end{aligned}
$$




$$
\begin{aligned}
& =\sum_{k \neq j}^{n} \int_{\omega_{k}\left(x_{k}\right)}^{\omega_{k}\left(x_{k}, x_{j}, \omega_{j}\left(x_{j}\right)\right)} d_{j k}(\tau, x, t) \beta_{j k}\left(x_{j k}, \omega_{j}\left(x_{j k}\right)\right) \frac{\left(a_{k} a_{j}\right)\left(x_{j k}, \omega_{j}\left(x_{j k}\right)\right)}{\partial_{3} \omega_{k}\left(x_{k}, x_{j k}, \omega_{j}\left(x_{j k}\right)\right)}\left(P D u^{l}\right)_{k}(\tau) d \tau \\
& =\sum_{k \neq j}^{n} \int_{\omega_{k}\left(x_{k}\right)}^{\omega_{k}\left(x_{k}, x_{j}, \omega_{j}\left(x_{j}\right)\right)} d_{j k}(\tau, x, t) \frac{\left(\beta_{j k} a_{k} a_{j}\right)\left(x_{j k}, \omega_{j}\left(x_{j k}\right)\right)}{\partial_{3} \omega_{k}\left(x_{k}, x_{j k}, \omega_{j}\left(x_{j k}\right)\right)} \sum_{s=1}^{n} p_{k s}\left(D u^{l}\right)_{s}\left(1-x_{s}, \tau\right) d \tau \\
& =\sum_{k \neq j}^{n} \int_{\omega_{k}\left(x_{k}\right)}^{\omega_{k}\left(x_{k}, x_{j}, \omega_{j}\left(x_{j}\right)\right)} d_{j k}(\tau, x, t) \frac{\left(\beta_{j k} a_{k} a_{j}\right)\left(x_{j k}, \omega_{j}\left(x_{j k}\right)\right)}{\partial_{3} \omega_{k}\left(x_{k}, x_{j k}, \omega_{j}\left(x_{j k}\right)\right)} \\
& \times \sum_{s=1}^{n} p_{k s} \int_{1-x_{s}}^{x_{s}} d_{s}\left(\xi, 1-x_{s}, \tau\right) \sum_{r \neq s}\left(b_{s r} u_{r}^{l}\right)\left(\xi, \omega_{s}\left(\xi, 1-x_{s}, \tau\right)\right) d \xi d \tau
\end{aligned}
$$

where

$$
d_{j k}(\tau, x, t)=d_{j}\left(x_{j k}(\tau, x, t), x, t\right) c_{k}\left(x_{k}, x_{j k}(\tau, x, t), \omega_{j}\left(x_{j k}(\tau, x, t)\right)\right)
$$

and $x_{j k}=x_{j k}(\tau, x, t)$ denotes the inverse map to $\xi \mapsto \tau=\omega_{k}\left(x_{k}, \xi, \omega_{j}(\xi)\right)$ for all $\xi$ such that $a_{k}\left(\xi, \omega_{j}(\xi)\right) \neq a_{j}\left(\xi, \omega_{j}(\xi)\right)$.

Write

$d_{j k s r}(\xi, \tau, x, t)=d_{j k}(\tau, x, t) \frac{\left(\beta_{j k} a_{k} a_{j}\right)\left(x_{j k}, \omega_{j}\left(x_{j k}\right)\right)}{\partial_{3} \omega_{k}\left(x_{k}, x_{j k}, \omega_{j}\left(x_{j k}\right)\right)} p_{k s} d_{s}\left(\xi, 1-x_{s}, \tau\right) b_{s r}\left(\xi, \omega_{s}\left(\xi, 1-x_{s}, \tau\right)\right)$.

Further we proceed with an arbitrary fixed summand in the right-hand side of (4.23). For definiteness, fix arbitrary $k \neq j, s$ in the range $m+1 \leq s \leq n$, and $r \neq s$. After applying Fubini's theorem to the corresponding summand in (4.23), it reads

$$
\begin{aligned}
\int_{0}^{1} & \int_{\omega_{k}\left(x_{k}\right)}^{\omega_{k}\left(x_{k}, x_{j}, \omega_{j}\left(x_{j}\right)\right)} d_{j k s r}(\xi, \tau, x, t) u_{r}^{l}\left(\xi, \omega_{s}(\xi, 0, \tau)\right) d \tau d \xi \\
& =\int_{0}^{1} \int_{\omega_{s}\left(\xi, 0, \omega_{k}\left(x_{k}\right)\right)}^{\omega_{s}\left(\xi, 0, \omega_{k}\left(x_{k}, x_{j}, \omega_{j}\left(x_{j}\right)\right)\right)} d_{j k s r}\left(\xi, \omega_{s}(0, \xi, \theta), x, t\right) \partial_{3} \omega_{s}(0, \xi, \theta) u_{r}^{l}(\xi, \theta) d \theta d \xi
\end{aligned}
$$

Here we used the change of variables $\tau \mapsto \theta=\omega_{s}(\xi, 0, \tau)$, that is, $\tau=\omega_{s}(0, \xi, \theta)$. Since

$$
\left|\omega_{s}\left(\xi, 0, \omega_{k}\left(x_{k}\right)\right)-\omega_{s}\left(\xi, 0, \omega_{k}\left(x_{k}, x_{j}, \omega_{j}\left(x_{j}\right)\right)\right)\right| \leq 2 d \text { for all }(x, t) \in \bar{\Pi},
$$

the $C\left(\bar{\Pi}_{\tau+2 d}^{\tau+2 d+\alpha}\right)^{n}$-norm of the right-hand side of (4.24) can be estimated from above by

$$
\begin{gathered}
2 d \max _{x, \xi \in[0,1]} \max _{t, \theta \in \mathbb{R}}\left|d_{j k s r}\left(\xi, \omega_{s}(0, \xi, \theta), x, t\right) \partial_{3} \omega_{s}(0, \xi, \theta)\right|_{\theta \in[\tau, \tau+2 d+\alpha]} \int_{0}^{1}\left|u_{r}^{l}(\xi, \theta)\right| d \xi \\
\leq K\left\|u_{r}^{l}\right\|_{C\left([\tau, \tau+2 d+\alpha], L^{2}(0,1)\right)},
\end{gathered}
$$

where $K$ is a constant independent of $u_{r}^{l}$. This implies the desired convergence for each summand in (4.23) and, therefore, for the whole $\left[D C P D u^{l}\right](x, t)$. This completes the proof of Claim 2.

The proof of $(4.16)$ for $\left[D(C P)^{i} D u^{l}\right](x, t)$ with $i=2, \ldots, k-1$ follows the same line, since the operator $C P$ is bounded. It follows that any $L^{2}$-generalized solution $u$ to the 
problem under consideration is a continuous function for all $t \geq \tau+T_{0}$, where $T_{0}=2 d$. Furthermore, the estimates (4.22) and (4.25) imply that

$$
\|u\|_{C\left(\bar{\Pi}_{\tau+T_{0}}^{\tau+T_{0}+\alpha}\right)^{n}} \leq K\|u\|_{C\left(\left[\tau, \tau+T_{0}+\alpha\right], L^{2}(0,1)\right)^{n}}
$$

where $K$ is a constant depending on $\alpha, a$ and $b$ but not on $\tau$. Using additionally the estimate (4.4), we come to the inequality

$$
\sup _{\tau+T_{0} \leq t \leq \tau+T_{0}+\alpha}\|U(t, \tau) \varphi\|_{C([0,1])^{n}} \leq K\|\varphi\|_{L^{2}(0,1)^{n}}, \quad t, \tau \in \mathbb{R}
$$

where $K$ is a constant independent of $\varphi$. Note that, given $c>0$, the constant $K$ can be chosen the same for all $b_{j k}$ such that $\max _{j, k}\left\|b_{j k}\right\|_{1}<c$. The proof of the lemma is complete.

The following smoothing result is proved in [17, Theorem 2.7].

Lemma 4.5 [17] Suppose that the conditions (2.4), (2.5), (2.8), (2.14), (2.15) are fulfilled. Then the evolution family $\{U(t, \tau)\}_{t \geq \tau}$ generated by the problem (2.7), (2.3) is smoothing from $Y_{0}$ to $C^{1}([0,1])^{n}$.

Theorem 2.5 follows from Lemmas 4.4 and 4.5. The smoothing time $T$, after which the $L^{2}$-generalized solution to the problem $(2.7),(2.3),(2.6)$ becomes $C^{1}$-smooth is equal to $T_{0}+T_{1}$, where $T_{0}$ is the smoothing time from $L^{2}$ - to $C$-regularity ensured by Lemma 4.4 and $T_{1}$ is the smoothing time from $C$ - to $C^{1}$-regularity ensured by [17, Theorem 2.7]. Furthermore, for given $\varphi \in L^{2}(0,1)^{n}$ and $\alpha>0$, the following bound is fulfilled:

$$
\sup _{\tau+T \leq t \leq \tau+T+\alpha}\|U(t, \tau) \varphi\|_{C^{1}([0,1])^{n}} \leq K\|\varphi\|_{L^{2}(0,1)^{n}}, \quad t, \tau \in \mathbb{R}
$$

where $K$ is a constant that depends on $\alpha, a, b$ and $p_{j k}(j, k \leq n)$ but not on $\tau$ and $\varphi$. Moreover, given $c>0$, the constant $K$ can be chosen the same for all $b_{j k}$ such that $\max _{j, k}\left\|b_{j k}\right\|_{1}<c$.

\section{Proof of the perturbation theorem}

\subsection{Abstract setting}

Let us write down the unperturbed and the perturbed problems (2.1), (2.6), (2.3) and (2.2), (2.6), (2.3), respectively, in the form of abstract evolution equations in the Hilbert space $L^{2}(0,1)^{n}$. To this end, denote $v(t)=\left(u_{1}(0, t), \ldots u_{m}(0, t), u_{m+1}(1, t), \ldots u_{n}(1, t)\right)$ and one-parameter families of operators $A(t)$ and $B(t)$ from $L^{2}(0,1)^{n}$ to $L^{2}(0,1)^{n}$ for each $t \in \mathbb{R}$, defined by

$$
\begin{aligned}
& (A(t) u)(x)=\left(-a(x, t) \frac{\partial}{\partial x}-b_{d}(x, t)\right) u, \\
& (B(t) u)(x)=(-\tilde{b}(x, t)) u
\end{aligned}
$$


where the domains of definition are given by

$$
\begin{aligned}
& D(A(t))=\left\{u \in L^{2}(0,1)^{n}: \partial_{x} u \in L^{2}(0,1)^{n}, v(t)=(P u)(t)\right\}, \\
& D(B(t))=L^{2}(0,1)^{n},
\end{aligned}
$$

for the operator $P$ given by (2.11). Note that $D(A(t)+B(t))=D(A(t))$.

In this notation, the unperturbed problem (2.1), (2.6), (2.3) reads

$$
\frac{d}{d t} u=A(t) u, \quad u(\tau)=\varphi \in L^{2}(0,1)^{n}
$$

while the perturbed problem (2.2), (2.6), (2.3) reads

$$
\frac{d}{d t} u=(A(t)+B(t)) u, \quad u(\tau)=\varphi \in L^{2}(0,1)^{n} .
$$

Accordingly to the above notation, $\{U(t, \tau)\}_{t \geq \tau}$ and $\{\tilde{U}(t, \tau)\}_{t \geq \tau}$ will denote evolution families on $L^{2}(0,1)^{n}$ generated by the problems (5.2) and (5.3), respectively.

\subsection{Proof of Theorem 2.7}

Part ( $\iota$ Consider the abstract formulations (5.2) and (5.3) of the unperturbed and perturbed problems (2.1), (2.6), (2.3) and (2.2), (2.6), (2.3), respectively. By Theorem 2.3, the problems (5.2) and (5.3) generate exponentially bounded evolution families $\{U(t, \tau)\}_{t \geq \tau}$ and $\{\tilde{U}(t, \tau)\}_{t \geq \tau}$, respectively. The condition (2.14) implies that

$$
U(t, \tau) \varphi=0 \quad \text { for all } t \geq \tau+d \quad \text { and } \varphi \in L^{2}(0,1)^{n}
$$

Set

$$
\beta=\max _{j, k}\left\|\tilde{b}_{j k}\right\|_{\infty}
$$

By Lemma (4.2), we have bounds

$$
\sup _{0 \leq t-\tau \leq d}\|U(t, \tau)\|_{\mathcal{L}\left(L^{2}(0,1)^{n}\right)} \leq C_{U}, \quad \sup _{0 \leq t-\tau \leq d}\|\tilde{U}(t, \tau)\|_{\mathcal{L}\left(L^{2}(0,1)^{n}\right)} \leq C_{\tilde{U}}
$$

for some positive constants $C_{U}$ and $C_{\tilde{U}}$ not depending on $\tau$.

Fix $\tau \in \mathbb{R}$ and $\varphi \in C_{0}^{\infty}([0,1])^{n}$. Then $U(t, \tau) \varphi$ and $\tilde{U}(t, \tau) \varphi$ are classical solutions to the problems (5.2) and (5.3), respectively. This allows us to apply the variation of constants formula (see, e.g. [27]), which gives us the equation

$$
\tilde{U}(t, \tau) \varphi=U(t, \tau) \varphi+\int_{\tau}^{t} U(t, s) B(s) \tilde{U}(s, \tau) \varphi d s \quad \text { for } \quad t \geq \tau
$$

where $B(t)$ is determined by (5.1). Our aim is to prove the bound (2.16). For $t>\tau+d$, the formula (5.6) reads

$$
\tilde{U}(t, \tau) \varphi=U(t, \tau) \varphi+\int_{\tau}^{t-d} U(t, s) B(s) \tilde{U}(s, \tau) \varphi d s+\int_{t-d}^{t} U(t, s) B(s) \tilde{U}(s, \tau) \varphi d s
$$


By (5.4), the first two summands in the right-hand side vanish, and we get

$$
\tilde{U}(t, \tau) \varphi=\int_{t-d}^{t} U(t, s) B(s) \tilde{U}(s, \tau) \varphi d s \quad \text { for } t>\tau+d
$$

Write $Z(t)=\|\tilde{U}(t, \tau) \varphi\|_{L^{2}(0,1)^{n}}$. Due to (5.5) and (5.7),

$$
\begin{gathered}
Z(t) \leq C_{\tilde{U}}\|\varphi\|_{L^{2}(0,1)^{n}} \quad \text { for } \quad \tau \leq t \leq \tau+d, \\
Z(t) \leq C_{U} \beta \int_{t-d}^{t} Z(s) d s \quad \text { for } \quad \tau+d<t
\end{gathered}
$$

Lemma 5.1 Suppose that the function $Z(t)$ fulfills the estimates (5.8), (5.9). Then for any $\gamma>0$ there exists $\varepsilon>0$ such that

$$
Z(t) \leq M e^{-\gamma(t-\tau)}\|\varphi\|_{L^{2}(0,1)^{n}} \quad \text { for } t \geq \tau
$$

for all $\beta \in(0, \varepsilon)$, where $M=C_{\tilde{U}} e^{\left(\gamma+C_{U} \varepsilon\right) d}$.

Proof. Fix an arbitrary $\gamma>0$. If $\tau<t \leq \tau+d$, then the desired estimate (5.10) follows from (5.8). Indeed,

$$
\begin{array}{r}
Z(t) \leq C_{\tilde{U}}\|\varphi\|_{L^{2}(0,1)^{n}}=C_{\tilde{U}} e^{\gamma(t-\tau)} e^{-\gamma(t-\tau)}\|\varphi\|_{L^{2}(0,1)^{n}} \leq M e^{-\gamma(t-\tau)}\|\varphi\|_{L^{2}(0,1)^{n}} \\
\text { for } 0<t-\tau \leq d .
\end{array}
$$

If $\tau+d<t \leq \tau+2 d$, then $\tau<t-d \leq \tau+d<t$ and, due to (5.8) and (5.9),

$$
\begin{aligned}
Z(t) & \leq C_{U} \beta \int_{t-d}^{t} Z(s) d s=C_{U} \beta\left[\int_{t-d}^{\tau+d} Z(s) d s+\int_{\tau+d}^{t} Z(s) d s\right] \\
& \leq C_{U} \beta\left[C_{\tilde{U}} d\|\varphi\|_{L^{2}(0,1)^{n}}+\int_{\tau+d}^{t} Z(s) d s\right] \\
& \leq C_{U} C_{\tilde{U}} \beta d\|\varphi\|_{L^{2}(0,1)^{n}}+C_{U} \beta \int_{\tau+d}^{t} Z(s) d s .
\end{aligned}
$$

By Gronwall's argument, we obtain

$$
Z(t) \leq C_{U} C_{\tilde{U}} \beta d e^{C_{U} \beta(t-\tau-d)}\|\varphi\|_{L^{2}(0,1)^{n}} \quad \text { for } \quad d<t-\tau \leq 2 d
$$

If $\tau+2 d<t \leq \tau+3 d$, then $\tau+d<t-d \leq \tau+2 d<t$ and, on the account of (5.9) and (5.11), we come to the inequality

$$
\begin{aligned}
Z(t) & \leq C_{U} \beta \int_{t-d}^{t} Z(s) d s=C_{U} \beta\left[\int_{t-d}^{\tau+2 d} Z(s) d s+\int_{\tau+2 d}^{t} Z(s) d s\right] \\
& \leq C_{\tilde{U}}\left(C_{U} \beta d\right)^{2} e^{C_{U} \beta d}\|\varphi\|_{L^{2}(0,1)^{n}}+C_{U} \beta \int_{\tau+2 d}^{t} Z(s) d s
\end{aligned}
$$


Again, the Gronwall's argument gives

$$
Z(t) \leq C_{\tilde{U}}\left(C_{U} \beta d\right)^{2} e^{C_{U} \beta(t-\tau-d)}\|\varphi\|_{L^{2}(0,1)^{n}} \quad \text { for } \quad 2 d<t-\tau \leq 3 d .
$$

Proceeding further by induction, on the $k$-th step we obtain an estimate for the function $Z(t)$, namely

$$
Z(t) \leq C_{\tilde{U}}\left(C_{U} \beta d\right)^{k} e^{C_{U} \beta(t-\tau-d)}\|\varphi\|_{L^{2}(0,1)^{n}} \quad \text { for } \quad k d<t-\tau \leq(k+1) d .
$$

Fix $\varepsilon$ to fulfill the equality

$$
\frac{1}{d} \log \left(C_{U} \varepsilon d\right)+C_{U} \varepsilon=-\gamma
$$

Note that $\log \left(C_{U} \varepsilon d\right)<0$. Since $t-\tau-d \leq k d$ and $\beta<\varepsilon$, we get

$$
\begin{aligned}
Z(t) & \leq C_{\tilde{U}} e^{k d \frac{1}{d} \log \left(C_{U} \varepsilon d\right)+C_{U} \varepsilon(t-\tau)}\|\varphi\|_{L^{2}(0,1)^{n}} \\
& \leq C_{\tilde{U}} e^{(t-\tau-d) \frac{1}{d} \log \left(C_{U} \varepsilon d\right)+C_{U} \varepsilon(t-\tau)}\|\varphi\|_{L^{2}(0,1)^{n}} \\
& =C_{\tilde{U}} e^{-\log \left(C_{U} \varepsilon d\right)} e^{-\gamma(t-\tau)}\|\varphi\|_{L^{2}(0,1)^{n}} \\
& =C_{\tilde{U}} e^{\left(\gamma+C_{U} \varepsilon\right) d} e^{-\gamma(t-\tau)}\|\varphi\|_{L^{2}(0,1)^{n}} \quad \text { for } \quad k d<t-\tau \leq(k+1) d,
\end{aligned}
$$

where the last equality holds by (5.12). Since $k \in \mathbb{N}$ is arbitrary, the estimate (5.10) follows. The proof of the lemma is complete.

Lemma 5.1 gives the estimate

$$
\|\tilde{U}(t, \tau) \varphi\|_{L^{2}(0,1)^{n}} \leq M e^{-\gamma(t-\tau)}\|\varphi\|_{L^{2}(0,1)^{n}} \quad \text { for } \quad t \geq \tau,
$$

for all $\varphi \in C_{0}^{\infty}([0,1])^{n}$. Since the space $C_{0}^{\infty}([0,1])^{n}$ is dense in $L^{2}(0,1)^{n}$, the same estimate is true for all $\varphi \in L^{2}(0,1)^{n}$. This entails $(2.16)$, as desired. The proof of part $(\iota)$ is complete.

Part $(\iota \iota)$ Fix an arbitrary $\gamma>0$. Let $\varepsilon$ be a positive real satisfying the estimate (2.16) for all $\tilde{b}_{j k}$ such that $\max _{j, k}\left\|\tilde{b}_{j k}\right\|_{\infty}<\varepsilon$.

Recall that all assumptions of Theorem 2.5 are stable with respect to certain perturbations of $b_{j k}$. Specifically, the condition (2.14) is stable, because it depends only on the diagonal part of $b$, while the perturbations involve only the non-diagonal part of $b$. The condition (2.15) is true by the corresponding assumption of Theorem $2.7(\iota \iota)$.

Therefore, by Theorem 2.5, $\tilde{U}(t, \tau)$ is smoothing from $L^{2}(0,1)^{n}$ to $C^{1}([0,1])^{n}$. More specifically, for any $\varphi \in L^{2}(0,1)^{n}$ the function $\tilde{U}(t, \tau) \varphi$ belongs to $C^{1}([0,1])^{n}$ whenever $t \geq \tau+T$ for some $T>0$. Furthermore, the estimate (4.26) implies that

$$
\|\tilde{U}(\tau+T, \tau) \varphi\|_{C^{1}([0,1])^{n}} \leq K_{0}\|\varphi\|_{L^{2}(0,1)^{n}} \quad \text { for all } \tau \in \mathbb{R}
$$

for some $K_{0} \geq 1$ not depending on $\varphi_{\tilde{b}}$ and $\tau$. Moreover, the constant $K_{0}$ can be chosen the same for all $\tilde{b}_{j k}$ such that $\max _{j, k}\left\|\tilde{b}_{j k}\right\|_{1}<\varepsilon$. 
Suppose that $\varphi \in C^{1}([0,1])^{n}$ satisfies the zero-order and the first-order compatibility conditions (4.1) and (4.2) for the perturbed problem (5.3). By Theorem 4.1, we have

$$
\|\tilde{U}(t, \tau) \varphi\|_{C^{1}([0,1])^{n}} \leq K_{T}\|\varphi\|_{C^{1}([0,1])^{n}} \quad \text { for all } \tau \leq t \leq \tau+2 T
$$

where $K_{T}=K_{1} e^{2 T \omega_{1}}>1$ for the constants $K_{1}$ and $\omega_{1}$ as in (4.3). Again, the constant $K_{1}$ and, hence $K_{T}$ can be chosen the same for all $\tilde{b}_{j k}$ such that $\max _{j, k}\left\|\tilde{b}_{j k}\right\|_{1}<\varepsilon$.

Fix an arbitrary $\tau \in \mathbb{R}$. To prove the estimate (2.17), it suffices to show that

$$
\|\tilde{U}(t, \tau) \varphi\|_{C^{1}([0,1])^{n}} \leq M_{1} e^{-\gamma(t-\tau)}\|\varphi\|_{L^{2}(0,1)^{n}} \quad \text { for all } t \geq \tau+2 T
$$

for all $\varphi \in L^{2}(0,1)^{n}$ and some $M_{1} \geq M$, where $M$ fulfills (2.16).

If $t \geq \tau+2 T$, then there is $k \geq 2$ such that $\tau+k T \leq t<\tau+(k+1) T$. Then $t-\tau-(k-1) T \leq 2 T$. Taking (5.14) into account, we see that

$$
\begin{aligned}
\|\tilde{U}(t, \tau) \varphi\|_{C^{1}([0,1])^{n}} & =\|\tilde{U}(t, \tau+(k-1) T) \tilde{U}(\tau+(k-1) T, \tau) \varphi\|_{C^{1}([0,1])^{n}} \\
& \leq K_{T}\|\tilde{U}(\tau+(k-1) T, \tau) \varphi\|_{C^{1}([0,1])^{n}}
\end{aligned}
$$

The estimates (5.13) and (2.16) imply that for $k \geq 2$

$$
\begin{aligned}
& \|\tilde{U}(\tau+(k-1) T, \tau) \varphi\|_{C^{1}([0,1])^{n}} \\
& \quad=\|\tilde{U}(\tau+(k-1) T, \tau+(k-2) T) \tilde{U}(\tau+(k-2) T, \tau) \varphi\|_{C^{1}([0,1])^{n}} \\
& \quad \leq K_{0}\|\tilde{U}(\tau+(k-2) T, \tau) \varphi\|_{L^{2}(0,1)^{n}} \\
& \quad \leq K_{0} M e^{-\gamma(k-2) T}\|\varphi\|_{L^{2}(0,1)^{n}}
\end{aligned}
$$

Finally, combining the estimates (5.15) and (5.16), we get

$$
\begin{aligned}
\|\tilde{U}(t, \tau) \varphi\|_{C^{1}([0,1])^{n}} \leq K_{T} K_{0} M e^{-\gamma(k-2) T}\|\varphi\|_{L^{2}(0,1)^{n}} & =K_{T} K_{0} M e^{3 \gamma T} e^{-\gamma(k+1) T}\|\varphi\|_{L^{2}(0,1)^{n}} \\
& \leq M_{1} e^{-\gamma(t-\tau)}\|\varphi\|_{L^{2}(0,1)^{n}}
\end{aligned}
$$

where $M_{1}=K_{T} K_{0} M e^{3 \gamma T}>M$ and $t \geq \tau+2 T$.

The proof of part $(\iota \iota)$ of Theorem 2.7 is complete.

\section{References}

[1] V.E. Abolinya, A.D. Myshkis, A mixed problem for an almost linear hyperbolic system on the plane, Matematicheskij Sbornik 50(92)(4) (1960), 423-442.

[2] G. Bastin, J.-M. Coron, Stability and Boundary Stabilization of 1-D Hyperbolic Systems, Progress in Nonlinear Differential Equations and Their Applications 88, Birkhäuser, 2016.

[3] A.V. Balakrishnan, On superstability of semigroups. Systems modelling and optimization, in: M.P.Polis et al. (Eds.), Proceedings of the 18th IFIP TC7 Conference on System Modelling and Optimization, CRC, Research Notes in Mathematics, Chapman and Hall (1999), 12-19. 
[4] A.V. Balakrishnan, Superstability of systems, Applied Mathematics and Computation 164(2) (2005), 321-326.

[5] J.-H. Chen, W.-Y. Lu, Perturbation of nilpotent semigroups and application to heat exchanger equations, Applied Mathematics Letters 24 (2011), 1698-1701.

[6] J.-M. Coron, G. Bastin, Dissipative boundary conditions for one-dimensional quasilinear hyperbolic systems: Lyapunov stability for the C1-norm, SIAM J. Control Optim. 53(3) (2015), 1464-1483.

[7] S. Cox, E. L. Zuazua, The rate at which energy decays in a string damped at one end, Indiana Univ. Math. J. 44(2) (1995), 545-573.

[8] D. Creutz, M. Mazo Jr., C. Preda, Superstability and finite time extinction for $C_{0^{-}}$ semigroups, (2013). E-print: https://arxiv.org/abs/0907.4812.

[9] L.E. Elsgolts, S.B. Norkin. An Introduction to the Theory and Application of Differential Equations with Deviating Arguments. Academic Press, New York, 1973.

[10] N.A. Ëltysheva, On qualitative properties of solutions to some hyperbolic systems on the plane, Matematicheskij Sbornik 135(2) (1988), 186-209.

[11] S.K. Godunov, Equations of Mathematical Physics, Moscow: Nauka, 2nd ed., 1979 (Russian).

[12] M. Gugat, Boundary feedback stabilization of the telegraph equation: Decay rates for vanishing damping term, Systems and Control Letters 66 (2014), 72-84.

[13] M. Gugat, Optimal Boundary Control and Boundary Stabilization of Hyperbolic Systems, Basel : Birkhäuser, 2015.

[14] E. Hille, R. Phillips, Functional analysis and semi-groups, Providence, 1957.

[15] I. Kmit, Classical solvability of nonlinear initial-boundary problems for first-order hyperbolic systems, Intern. J. Dynamic Systems Different. Equat. 1(3) (2008), 191195.

[16] I. Kmit, Smoothing effect and Fredholm property for first order hyperbolic PDEs, In: Operator Theory: Advances and Applications, Basel: Birkhäuser 231 (2013), 219-238.

[17] I. Kmit, Smoothing solutions to initial-boundary problems for first-order hyperbolic systems, Applicable Analysis 90(11) (2011), 1609-1634.

[18] I. Kmit, G. Hörmann, Systems with singular non-local boundary conditions: Reflection of singularities and delta waves, J. Anal. Appl. 20(3) (2001), 637-659.

[19] V. Komornik, Rapid boundary stabilization of the wave equation, SIAM J. Control Optim. 29 (1991), 197-208. 
[20] M.G. Krein, I.C. Gohberg, Theory and applications of Volterra operators in Hilbert space, American Math Society, 1970.

[21] M.M. Lavrent'ev Jr., N.A. Lyul'ko, Increasing smoothness of solutions to some hyperbolic problems, Siberian Math. J. 38(1) (1997), 92-105.

[22] N.A. Lyul'ko, Increasing smoothness of solutions to a hyperbolic system on the plane with delay in the boundary conditions. Siberian Math. J. 49(6) (2008), 1333-1350.

[23] G. Lumer, On the growth of the resolvents for an explicit class of superstable semigroups. Ulmer Seminare uber Funktionalanalysis and Differentialgleichungen, Appl. Analysis, Univ. Ulm 6 (2002), 253-258.

[24] A. Majda, Disappearing solutions for the dissipative wave equation, Indiana Univ. Math. J. 24 (1975), 1119-1133.

[25] L. Pavel, Classical solutions in Sobolev spaces for a class of hyperbolic Lotka-Volterra systems, SIAM J. Control Optim. 51(3) (2013), 2132-2151.

[26] L. Pavel, L. Chang, Lyapunov-based boundary control for a class of hyperbolic LotkaVolterra systems, IEEE Trans. Automat. Control 7 (2012), 701-714.

[27] A. Pazy, Semigroups of operators and applications to partial differential equations, Springer-Verlag, Berlin, 1983.

[28] V. Perrollaz, L. Rosier, Finite-Time Stabilization of $2 \times 2$ Hyperbolic Systems on Tree-Shaped Networks, SIAM Journal on Control and Optimization 52(1) (2014), $143-163$.

[29] F. Räbiger and M. Wolff, Superstable semigroups of operators, Indagationes Mathematicae 6 (1995), 481-494.

[30] R.K. Romanovskiy, E.V. Vorobyova, E.N. Stratilatova, The Riemann method for hyperbolic systems, Novosibirsk: Nauka, 2007 (Russian).

[31] Y. Shang, D. Liu, G. Xu, Super-stability and the spectrum of one-dimensional wave equations on general feedback controlled networks, IMA Journal of Mathematical Control and Information 31(1) (2014), 73-99.

[32] F.E. Udwadia, Boundary control, quiet boundaries, super-stability and superinstability, Applied Mathematics and Computation 164(2) (2005), 327-349.

[33] F.E. Udwadia, On the longitudinal vibrations of a bar with viscous boundaries: Super-stability, super-instability and loss damping, Intern. J. of Engineering Science 50(1) (2012), 79-100.

[34] T.I. Zelenyak, On stationary solutions of mixed problems relating to the study of certain chemical processes, Differ. Equations 2(2) (1966), 205-213. 
[35] T.I. Zelenyak, On the question of stability of mixed problems for a quasi-linear equation (Russian), Differ. Equations 3(1) (1967), 19-29. 